\title{
A NEW RAYTRACER FOR MODELING AU-SCALE IMAGING OF LINES FROM PROTOPLANETARY DISKS
}

\author{
Klaus M. Pontoppidan ${ }^{1,3}$, Rowin Meijerink ${ }^{1}$, Cornelis P. Dullemond ${ }^{2}$, and Geoffrey A. Blake ${ }^{1}$ \\ ${ }^{1}$ California Institute of Technology, Division of Geological and Planetary Sciences, MS 150-21, Pasadena, CA 91125, USA; pontoppi@gps.caltech.edu \\ ${ }^{2}$ Max-Planck-Institut für Astronomie, Heidelberg, Königstuhl 17, 69117 Heidelberg, Germany \\ Received 2009 July 2; accepted 2009 September 8; published 2009 October 2
}

\begin{abstract}
The material that formed the present-day solar system originated in feeding zones in the inner solar nebula located at distances within $\sim 20 \mathrm{AU}$ from the Sun, known as the planet-forming zone. Meteoritic and cometary material contain abundant evidence for the presence of a rich and active chemistry in the planet-forming zone during the gas-rich phase of solar system formation. It is a natural conjecture that analogs can be found among the zoo of protoplanetary disks around nearby young stars. The study of the chemistry and dynamics of planet formation requires: (1) tracers of dense gas at 100-1000 K and (2) imaging capabilities of such tracers with 5-100 mas (0.5-20 AU) resolution, corresponding to the planet-forming zone at the distance of the closest star-forming regions. Recognizing that the rich infrared $(2-200 \mu \mathrm{m})$ molecular spectrum recently discovered to be common in protoplanetary disks represents such a tracer, we present a new general ray-tracing code, RADLite, that is optimized for producing infrared line spectra and images from axisymmetric structures. RADLite can consistently deal with a wide range of velocity gradients, such as those typical for the inner regions of protoplanetary disks. The code is intended as a back-end for chemical and excitation codes, and can rapidly produce spectra of thousands of lines for grids of models for comparison with observations. Such radiative transfer tools will be crucial for constraining both the structure and chemistry of planet-forming regions, including data from current infrared imaging spectrometers and extending to the Atacama Large Millimeter Array and the next generation of Extremely Large Telescopes, the James Webb Space Telescope and beyond.
\end{abstract}

Key words: astrochemistry - planetary systems: protoplanetary disks - radiative transfer - techniques: high angular resolution

Online-only material: color figures

\section{INTRODUCTION}

The least processed components of the solar system-comets, asteroids, and Kuiper Belt Objects (KBOs) — are witnesses to an active solar nebula endowed with a rich chemistry. Much of the formation and early evolution of the solar system took place at small distances (perhaps within $20 \mathrm{AU}$ ) from the Sun where densities were high. Only at later stages did the solar system expand to its present size (as defined by the Kuiper Belt and Oort Cloud), probably due to dynamical scattering processes involving planetesimals. It is thought that the KBOs were formed within $35 \mathrm{AU}$ (Levison \& Morbidelli 2003), while the planets were all formed within $20 \mathrm{AU}$, Jupiter and Saturn within $10 \mathrm{AU}$ (Tsiganis et al. 2005). Oort Cloud comets are thought to have formed in the 5-10 AU region, and are composed of processed material that may have passed much closer to the Sun (Wooden et al. 2007). One group of important tracers of the solar nebula is primitive meteorites, including carbonaceous chondrites, since these can be brought into a laboratory setting and studied in great detail. Generally, such bodies formed in a relatively narrow range of radii from 2 to $4 \mathrm{AU}$ (Morbidelli et al. 2002).

While the remnants of the formation of the solar system tell us an exciting story, sometimes in great detail, they can never paint a full picture of planet formation in general because the evidence is ancient and hence often contaminated by processing and, perhaps more importantly, the solar system is only one instance of a process that appears to be highly stochastic resulting in a

\footnotetext{
3 Hubble Fellow
}

wide range of planetary configurations (Udry \& Santos 2007). For these reasons, the region spanning $R=0.1-20 \mathrm{AU}$ in accretion disks around young stars can be considered as the primary target for observational studies of exo-planet formation. The part of a protoplanetary disk (pp) within this range of radii is often putatively referred to as the planet-forming region.

Given the angular sizes (5-100 mas) and surface temperatures $(100-2000 \mathrm{~K})$ of the planet-forming regions around T Tauri stars in the nearest star-forming regions, they are most readily traced by molecular and atomic lines in the infrared-roughly 2-200 $\mu \mathrm{m}$. Over the next decade, the mapping of the chemistry and structure of the planet-forming regions within hundreds of nearby protoplanetary disks, using infrared gas-phase signatures, can be expected to provide a critical complement to the study of mature planetary systems around main-sequence stars.

The first steps in this direction have already been taken, and an increasing number of infrared gas-phase tracers have been identified. The CO fundamental $(v=1-0)$ rovibrational band at $4.7 \mu \mathrm{m}$ is arguably the most accessible infrared molecular band. It has been used extensively to trace gas in protoplanetary disks (Najita et al. 2003; Blake \& Boogert 2004; Salyk et al. 2007), and CO fundamental line emission from disks has in a few cases been directly imaged using $8-10$ class telescopes with adaptive optics (Goto et al. 2006; Pontoppidan et al. 2008; van der Plas et al. 2009). Recently, Spitzer spectroscopy has demonstrated that the 10-36 $\mu \mathrm{m}$ range of disks is covered in molecular and atomic emission lines (Carr \& Najita 2008; Salyk et al. 2008), dominated by $\mathrm{H}_{2} \mathrm{O}, \mathrm{OH}, \mathrm{HCN}, \mathrm{C}_{2} \mathrm{H}_{2}$, and $\mathrm{CO}_{2}$, but also including important atomic diagnostic lines such as the [Ne II] transition at $12.81 \mu \mathrm{m}$ (Pascucci et al. 2007; Lahuis et al. 
2007; Najita et al. 2009). In the visible, the [O I] 6300 Å line has also been used as an inner disk tracer of $\mathrm{OH}$ (Acke et al. 2005; Fedele et al. 2008; van der Plas et al. 2008).

Among the central goals for kinematic imaging of planetforming zones will be (1) a search for protoplanets and their dynamical interaction with their parent disks, (2) a search for chemical processes inferred for the solar nebula, and (3) a general investigation of chemistry in planet-forming regions to better understand the initial conditions for planet formation. Key questions that can be answered include: if and how is water transported inside the snow line in protoplanetary disks, as was required to form the Earth's oceans (see Encrenaz 2008, for a review)? Is it possible to draw direct connections between protoplanetary disks and the solar nebula chemistry inferred from primitive meteorites (Lyons \& Young 2005; Smith et al. 2009)? Are the inner holes and gaps observed in some protoplanetary disks associated with planet formation (Strom et al. 1989; Calvet et al. 2002; Lada et al. 2006; Brown et al. 2007)? Can protoplanets be detected dynamically through their interactions with the gaseous component of their parent protoplanetary disks? If so, what are their radial and mass distributions and how do these compare to the distributions of the population of mature exo-planets? Massive protoplanets are expected to form their own circumplanetary accretion disks (Canup \& Ward 2002; Machida et al. 2008; Ayliffe \& Bate 2009) - such as the disk that formed the Jovian moon system. Is it possible to detect circumplanetary disks and use their kinematic signature to confirm the presence of a protoplanet?

In this paper, we present a new two-dimensional ray-tracing code, called RADLite ${ }^{4}$, that is written specifically to rapidly and efficiently render images and spectra of lines in the infrared, such as those tracing the planet-forming region, for a full gas+dust two-dimensional density structure. Note, however, that there is no fundamental reason why the code cannot also be applied to lines in the visible or (sub)millimeter region. Most observational studies of infrared lines use very simple models, such as slab geometries or single layer thin disk calculations, to simulate lines from a growing database of high-quality infrared line observations (e.g., Najita et al. 2003; Blake \& Boogert 2004; Thi \& Bik 2005; Brittain et al. 2007; Pontoppidan et al. 2008). This is in sharp contrast to the relatively advanced stage of radiative transfer modeling of rotational lines in the millimeter regime (Hogerheijde \& van der Tak 2000; Pavlyuchenkov et al. 2007). With RADLite, we adopt a new philosophy: recognizing the very high complexity of inner disk chemistry and line excitation, we decouple these steps from the simulation and fitting of observational data. Because RADLite is fast, a user is able to calculate grids of models to determine best fitting absolute level population distributions in axisymmetric geometries. With these in hand, the application of, and comparison to, chemistry and excitation calculations becomes a much simpler task. An example of such an application to the mid-infrared lines of water vapor from protoplanetary disks is presented in Meijerink et al. (2009). Here, the basic code is described in Section 2, and the simulation of telescopic line imaging with the future generation of Extremely Large Telescopes (ELTs) is described in Section 3.

As a demonstration, we apply RADLite to two examples simulating ground-based high-resolution (spectral and spatial) observations in Section 4: CO rovibrational emission from the fundamental $(v=1-0)$ band at $4.7 \mu \mathrm{m}$ (Section 4.2) and rotational water emission lines in the $N$ band at 10-14 $\mu \mathrm{m}$

\footnotetext{
4 RADLite is currently available on a collaborative basis, with a wider circulation anticipated in the near future.
}

(Section 4.3). These examples are used to demonstrate how RADLite simulates four common spectroscopic observables: line profiles, echellograms (also known as position-velocity diagrams or two-dimensional slit images), spectro-astrometry and integral field unit (IFU) image cubes. A fifth observable, interferometric visibilities, is not explicitly simulated, but can easily be constructed from existing RADLite products, should the need arise.

It is concluded, given that molecular lines in the near- to midinfrared wavelength region are prime tracers of the dynamics and chemistry of the planet-forming region, that the next generation of ground-based ELTs will be ideal for producing resolved line images. This requires an imaging spectrometer with resolving powers of $R=\lambda / \Delta \lambda=50,000-100,000$. The Atacama Large Millimeter Array (ALMA), currently under construction, is also capable of high-resolution molecular line imaging. This facility offers a tremendous improvement over previous millimeter arrays, and has sufficient spatial resolution to provide images with 1-2 AU resolution of the planetforming region in the continuum (Wolf \& D'Angelo 2005). For line observations, the ALMA will reach spatial resolutions of $\sim 10-20 \mathrm{AU}$, or somewhat larger radii than those traced by infrared lines (Semenov et al. 2008). Further, some molecular species, such as the symmetric rotators $\mathrm{CH}_{4}, \mathrm{CO}_{2}$, and $\mathrm{C}_{2} \mathrm{H}_{2}$, available in the infrared cannot be reached with the ALMA and vice versa. Perhaps the most notable example of a molecule generally considered to be an infrared or space-based tracer is $\mathrm{H}_{2}^{16} \mathrm{O}$. Therefore, future observations of infrared lines tracing the inner disk (0.1-10 AU) will be highly complementary to the (sub)millimeter tracers to be imaged by the ALMA, and powerful synergies between these facilities can be anticipated.

\section{RADIATIVE TRANSFER MODEL}

\subsection{A Raytracer for Infrared Lines from Protoplanetary Disks}

RADLite is a general ray-tracing code that has been optimized for use with infrared lines in axisymmetric density distributions. It is intended to be used as a back-end to dust radiative transfer and line excitation calculations. The continuum calculation should provide a dust temperature and the source function due to scattered photons in each grid point, as implemented in the continuum Monte Carlo code RADMC (Dullemond \& Dominik 2004), while the line excitation calculation should provide level populations for a given molecular or atomic species. This construction is designed to take advantage of the growing availability of multi-dimensional line radiative transfer codes that need a tool to simulate observational data (e.g., Woitke et al. 2009). In our implementation, RADLite uses RADMC for the dust radiative transfer, which is recommended for use with RADLite. For the line excitation beta3D (Poelman \& Spaans 2005; Meijerink et al. 2008) is used in a 1D+1D configuration, but RADLite can interface with any axisymmetric line excitation code. RADLite does not assume that the grid spacing is regular in the radial or angular direction. If the input chemical and/or excitation model has a fine gridding over a certain range of the angular coordinate, $\theta$, perhaps to adequately sample steep gradients in abundance or temperature in the surface layer of a disk, RADLite can simply use that grid directly. If the user does not wish to carry out a detailed balance calculation for the level populations, RADLite can also render images and spectra assuming that the level populations are those given by local thermodynamic equilibrium (LTE). In all cases, RADLite accepts molecular parameter input (levels energies, statistical 
weights, and Einstein A values) directly from the HITRAN database (Rothman et al. 2005) or in the format of the Leiden Atomic and Molecular Database (LAMDA; Schöier et al. 2005). In the following, we use parameters from HITRAN.

RADLite is based on the "long characteristics" (LC) method of integration of the transfer equation in polar coordinates presented in Dullemond \& Turolla (2000). LC integrations are performed by constructing a large number of parallel rays passing through a given density structure at different impact parameters. Each ray is then divided into a number of ray elements defined by the boundaries of the density structure grid elements. The formal transfer equation is integrated across each ray element, starting from infinity. For a dust continuum source function, this integration usually only requires that the density and temperature structures are well approximated by a linear function across the ray element. RADLite includes the line emissivity function, $j_{v}^{l}$, and opacity, $\alpha_{v}^{l}$, terms in the transfer equation:

$$
\frac{d I_{v}}{d s}=\left[\alpha_{v}^{c}(R, \theta)+\alpha_{v}^{l}(R, \theta, \bar{v})\right]\left[S_{v}(R, \theta, \bar{v})-I_{\nu}\right],
$$

where the source function is

$$
S_{v}=\frac{j_{v}^{c}(R, \theta)+j_{v}^{l}(R, \theta, \bar{v})}{\alpha_{v}^{c}(R, \theta)+\alpha_{v}^{l}(R, \theta, \bar{v})},
$$

and $\bar{v}$ is the macroscopic velocity projected on the ray ( $R$ and $\theta$ are the polar coordinates of the grid).

The line source and opacity functions can vary on much smaller spatial scales than the continuum functions. Such cases typically result from a narrow intrinsic line profile coupled with large macroscopic velocity gradients. For a one-dimensional model (radial motion only) with a monotonic velocity gradient, the problem could be solved by applying the Sobolov, or large velocity gradient (LVG), approximation. On a general axisymmetric grid, the LVG approximation cannot be assumed to hold, a priori, because a line may interact with itself at several different locations along a ray (an example of a situation where this happens is discussed in Section 4.2). For an LC integration that normally evaluates the integral of the transfer equation at the boundaries of the model grid, a small intrinsic line broadening relative to the global velocity gradient can result in such rapid changes of the source function that these are entirely contained within the endpoints of a ray element. This situation is illustrated in Figure 1. The problem is essentially one of undersampling, and can be resolved if the disk model is defined on a very fine grid. However, such a brute force solution generally results in a great loss of computational efficiency because the vast majority of integration steps do not interact with a line. The result is very long processing times-more than 10 minutes per line on current workstations. Since an important goal of RADLite is to be able to render grids of models, with each model potentially containing thousands of lines (e.g., the forest of water lines in the infrared), such inefficient raytracing would be unacceptable.

How bad is this undersampling problem? To explain the viscous timescales of typical PP disks, the turbulent velocities are predicted to be small (Hartmann et al. 1998). For low turbulence, intrinsic line widths are predicted to be dominated by thermal broadening, which in some cold regions, such as the disk mid-plane, can be as low as tens of $\mathrm{m} \mathrm{s}^{-1}$. In the inner disk, and for typical size grids used for the rendering of continuum images and spectra, the jump in velocities, $\Delta v$, can be of order a few up to $10 \mathrm{~km} \mathrm{~s}^{-1}$, typically at $\sim 0.1 \mathrm{AU}$ for a T

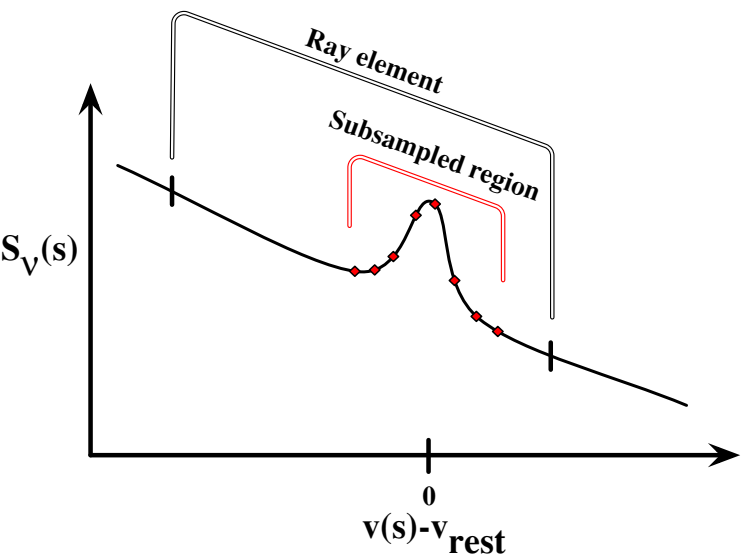

Figure 1. Sketch of the RADLite ray element subsampling scheme. The illustration shows the line and continuum source $S_{v}$, as a function of radial velocity $v(s)$, which in turn is a function of location $s$ along the ray. Normally, the integral of the transfer equation is only evaluated at each end of the ray element, as indicated in the figure. However, lines with narrow local broadening may be completely missed by the integration. In such cases, the line is localized within the ray element and the integrand evaluated in a sufficient number of points across the line.

(A color version of this figure is available in the online journal.)

Tauri star. In other words, the length, in velocity space, of a ray element may be 1-2 orders of magnitude larger than the width of the line. In these cases, the contribution from the line will simply not be included in the integration, causing an erroneous surface brightness along that ray. RADLite solves the problem by using an optimal form of grid refinement, while still allowing the global density structure to be defined on a relatively coarse spatial grid - typically one designed for dust radiative transfer. For each integration step across a given ray element, the code checks for line crossings by assuming that the velocity projected along the ray is a smooth, linear function. If a line crosses the ray within a ray element, a subdivision of the element is done in such a way that the line is integrated with an optimal number of samples (usually about 5 per FWHM of the line). The physical parameters, density, level population, etc., are calculated within a ray element using a simple linear interpolation between the values evaluated where the ray crosses the cell boundary. This approach is illustrated in Figure 1.

\subsection{Ray Configuration}

The optimal sampling along a ray takes care of undersampling in one dimension. However, proper rendering of line spectra and image cubes also requires that the set of rays is arranged with a sufficiently fine spacing. For instance, in the case of a protoplanetary disk, infrared lines arise from a thin surface layer. This gives rise to the line emission being formed in a thin band along the isovelocity curve in the intensity image at a given frequency or line velocity (see Figure 2). The isovelocity curve is a curve along which the projected velocity of the emitting disk surface is constant. A disk has two surfaces, and each surface will produce line emission along an isovelocity curve. If the disk is optically thick to continuum photons, only one isovelocity curve will be seen because dust blocks the observers view of the other surface. For illustrative purposes, the line in Figure 2 is chosen such that the disk has an optical depth close to unity, allowing the other surface to be seen. The smaller the intrinsic line broadening, the narrower the emission band. If the image is not sufficiently spatially resolved (i.e., if the pixels are not small 


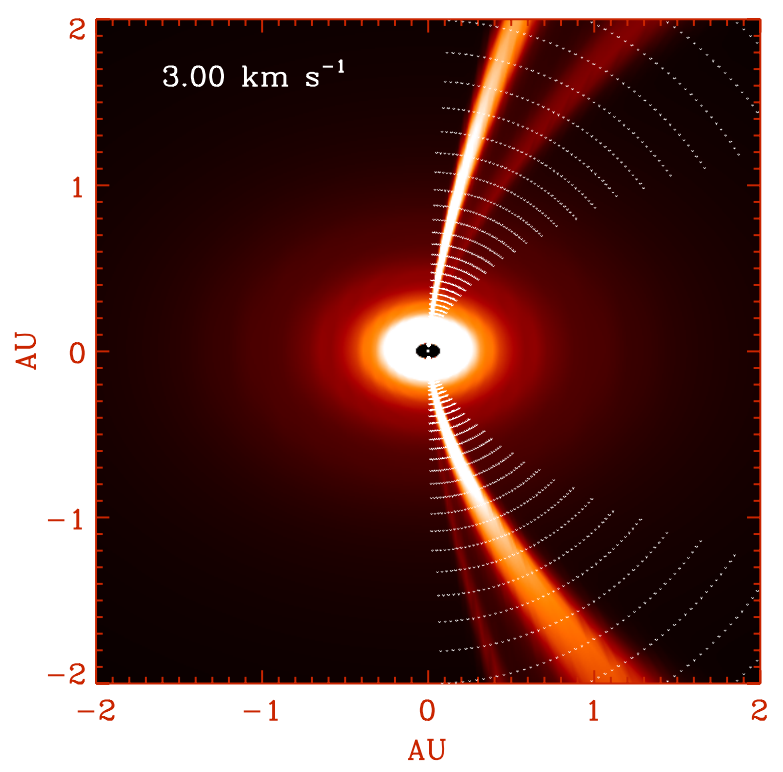

Figure 2. Unconvolved intensity image $(I(x, y, v))$ at $3 \mathrm{~km} \mathrm{~s}^{-1}$ for the $108 \mu \mathrm{m}$ $2_{2} 1 \rightarrow 1_{10} \mathrm{H}_{2} \mathrm{O}$ line for the fiducial protoplanetary disk model viewed at an inclination of $45^{\circ}$. For illustrative purposes, the maximal water abundance is lowered to $10^{-8}$ per hydrogen nucleus to keep the line optically thin. Due to the relatively long wavelength of this line, the disk is also has a dust optical depth of $\sim 1$, allowing the line image to show emission from both surfaces of the disk, resulting in line emission along two isovelocity contours. The white points indicate rays in which RADLite has detected line interactions. The far side of the disk is toward the top of the image.

(A color version of this figure is available in the online journal.)

enough), the thin isovelocity band may easily be missed, again resulting in severe underestimates of line fluxes. The RADLite strategy to deal with this, without sacrificing efficiency, is to define a large number of closely spaced rays, but to only integrate those that actually interact with the line. All other rays are considered "continuum rays," and are integrated only once, assuming that the continuum emission does not change with frequency across the line. The rays are arranged in concentric circles with 150-600 rays per circle. The circles are spaced according to the same radial logarithmic grid of the density structure. In the inner disk, additional 50-100 ray circles are added spanning inward to the stellar surface in order to properly sample the inner rim. Line spectra are calculated by a simple surface integral; the intensities of each ray circle are averaged, and the total intensity calculated by a one-dimensional radial integral of the circle averages. The star is included as a special central ray. The user can specify whether to use a blackbody or a more complex stellar model. A typical ray configuration for optimal line rendering is shown in Figure 2.

The user can modify the sampling of the rays, depending on the application. For imaging, typically more rays are needed to avoid aliasing effects, i.e., artifacts caused by interpolating undersampled data from a circular grid to a rectangular grid. RADLite outputs the circular images, i.e., the ray configuration and the associated intensities. The post-processing IDL script can then be used to resample the image on a rectangular mesh, with an arbitrary spatial sampling. The resampling uses TRIGRID, a bilinear interpolation scheme in IDL. As with all interpolation schemes, undersampling can be a problem, but the user can verify that the flux is conserved by comparing to the surface integral of the original circular image. Typical interpolation errors are of order $1 \%$ in the results presented here.

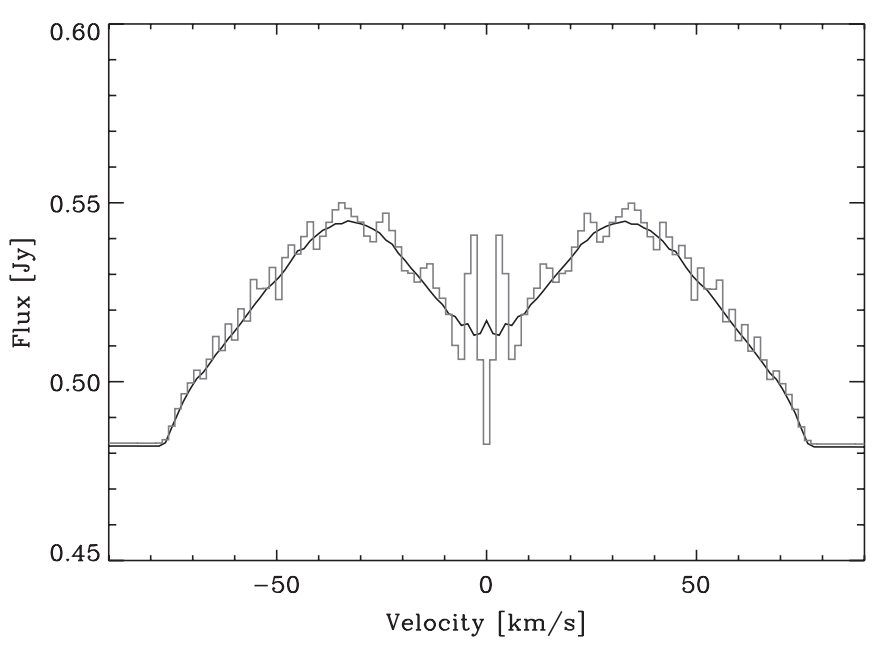

Figure 3. Comparison of a rovibrational CO line rendered with (black curve) and without (gray histogram) the optimal gridding for the same computational time. This particular model with the optimal gridding turned off could be made to produce the same result as the optimal model, with a choice of a very fine global grid and a large number of rays, but at the cost of about a factor $10 \mathrm{in}$ computational time.

Including the enhancements discussed above, RADLite renders a spectrum and image-velocity cube of a line at $3 \mathrm{~km} \mathrm{~s}^{-1}$ resolution on a single $3 \mathrm{GHz}$ Intel Xeon processor core in 15$30 \mathrm{~s}$. Line spectra can be rendered using $\sim 50,000$ rays, while high-quality images may require up to 300,000 rays.

\subsection{Benchmark}

There is currently no appropriate benchmark of RADLite, given the lack of other codes covering the same parameter space. However, it was confirmed that RADLite reproduces the result of RAYTRACE, the well-tested continuum raytracer associated with RADMC, to a fraction of a percent. This ensures that the dust radiative transfer is carried out correctly. In Figure 3, we compare a line calculated with and without the RADLite subgridding and selective ray calculation. The ray gridding is adjusted such that the two lines take the same number of CPU cycles to render. In practice, the difference between results achieved with the RADLite subgridding, as compared to brute force calculations, varies widely with the line and the underlying disk structure. Furthermore, RADLite reproduces the results of the toy model fit to the SR 21 disk from Pontoppidan et al. (2008; see Section 4.2).

Finally, given that a significant volume of radiative transfer work is available for (sub)millimeter lines, a model was constructed to render the rotational lines of ${ }^{13} \mathrm{CO}(3-2)$ and $\mathrm{HCN}$ (4-3) for a disk with the same parameters as those used by van Zadelhoff et al. (2001) for the T Tauri star LkCa 15 based on a two-layer passive disk model (Chiang \& Goldreich 1997). The modeled line spectra are shown in Figure 4. The main differences are that our model is based on a full two-dimensional dust model and assumes LTE level populations, while the van Zadelhoff et al. (2001) does not. It can be noted that the critical densities of the modeled lines are $\sim 3.5 \times 10^{4} \mathrm{~cm}^{-3}$ and $\sim 1.2 \times 10^{8} \mathrm{~cm}^{-3}$, so it is more likely that HCN is out of LTE than CO (the mid-plane of the van Zadelhoff et al. 2001 disk has densities lower than the HCN (4-3) critical density beyond $\sim 150 \mathrm{AU})$. This is reflected by a stronger RADLite LTE line. RADLite reproduces in detail the line strength and shape of the ${ }^{13} \mathrm{CO}(3-2)$ transition, which is known to be in LTE throughout the disk mid-plane. 

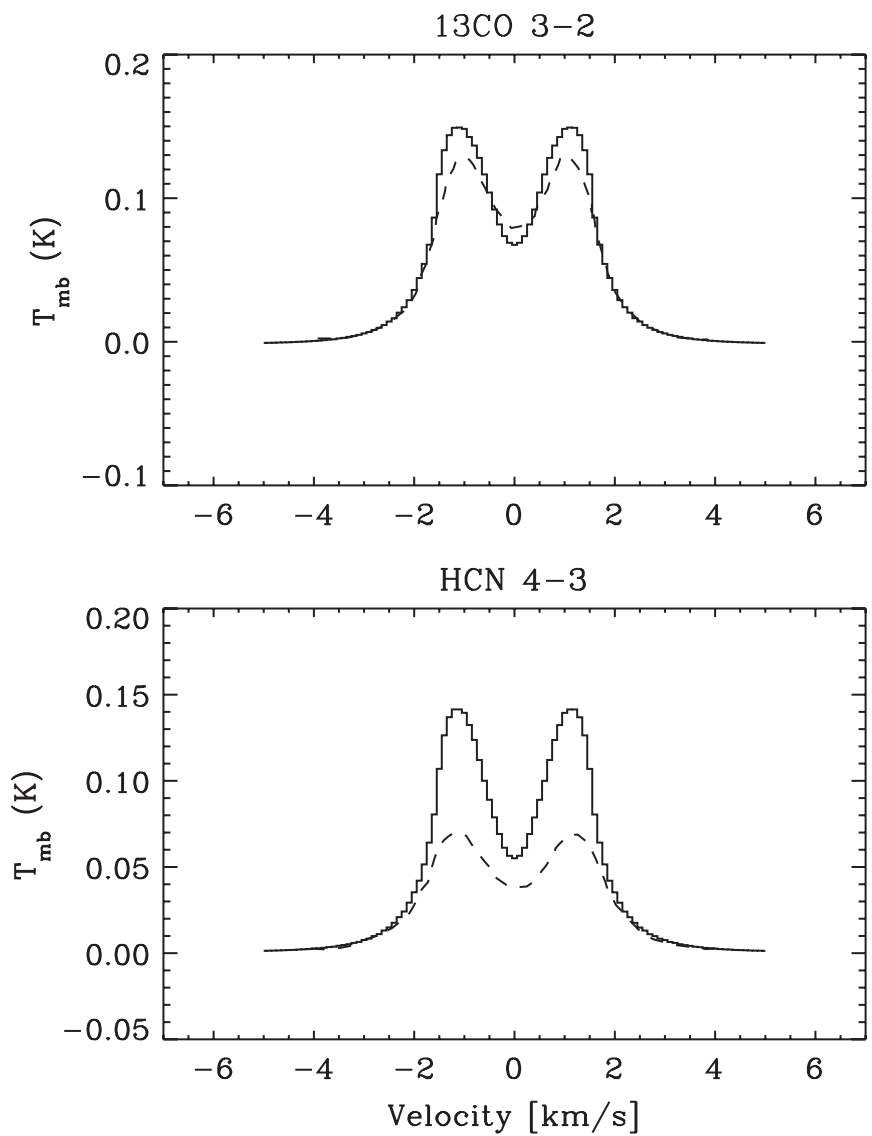

Figure 4. Continuum-subtracted RADLite LTE renderings (solid curves) of the rotational ${ }^{13} \mathrm{CO}(3-2)$ and $\mathrm{HCN}(4-3)$ lines for a disk model designed to be as close as possible to that implemented for LkCa 15 by van Zadelhoff et al. (2001, dashed curves). The difference in strengths of the $\mathrm{HCN}$ lines can be explained by subthermal excitation in the outer disk in the van Zadelhoff et al. (2001) model, given the high critical density of this transition $\left(1.2 \times 10^{8} \mathrm{~cm}^{-3}\right)$.

\subsection{Caveats}

While RADLite is not limited to a particular geometry or velocity field, apart from the strict requirement that the density structure is limited to two-dimensional polar coordinates, a number of caveats still apply. One property of the disk emission that is not modeled in a fully self-consistent manner is scattering. Dust scattering of continuum photons is included, but is assumed to be isotropic. Energy emitted in the lines, however, is not able to scatter on dust. Including this would require a Monte Carlo approach, which would be extremely time consumingespecially if a large number of lines are to be simulated. Lines are also rendered assuming that there are no interactions with other lines. We find that this is generally a very good approximation for protoplanetary disks. In this case, line interactions will only happen when isovelocity contours overlap. For a Keplerian disk, this only occurs when the difference in line rest frequencies between overlapping lines is of the order of the local line broadening or smaller, i.e., $\lesssim 1 \mathrm{~km} \mathrm{~s}^{-1}$. In the general case, for different velocity fields and molecular species, it is possible to find cases where line overlaps are a problem. One example is the $Q$-branches of the mid-infrared $\mathrm{HCN}, \mathrm{CO}_{2}$, and $\mathrm{C}_{2} \mathrm{H}_{2}$ bending rovibrational bands. The shift between transitions of different isotopologues (e.g., $\mathrm{H}_{2}^{16} \mathrm{O}$ to $\mathrm{H}_{2}^{18} \mathrm{O}$ ) is generally too large to cause any problems with line overlaps.

\section{SIMULATING OBSERVATIONS}

The primary purpose of RADLite is to simulate observations, and the package is therefore equipped with simple post-processing scripts that can, given parameters for a virtual telescope, convert ideal images and spectra to three typical observational products: single-slit spectra, astrometric spectra, and image cubes (the last as would be observed with an idealized IFU). However, it is foreseen that users typically will want to supply their own telescopic models to suit some required level of complexity of the telescope simulation. For this reason, this step is not considered as an integral part of RADLite, but is provided as an optional post-processing IDL script. In the following, the telescope simulation that is currently used is presented. Tools that are not yet developed include visibility calculations for current and future infrared interferometers, such as VLT-MATISSE (Lopez et al. 2008).

\subsection{Telescopic Observables}

First, the way RADLite treats the basic observables, image cubes, echellograms, line profiles, and spectro-astrometry, is described. The simulations assume that observations are made with grating spectrometers (as is the case for almost all current midinfrared astronomical instrumentation). A grating spectrometer images a spectrally dispersed source on a two-dimensional detector after the image of the source (a point-spread function (PSF) for a point source) has been truncated by a passage through a (approximately rectangular) slit with a length and a width, measured in angular units. This configuration results in a spectral image or echellogram that has a spatial dimension (along the slit) and a spectral dimension (across the slit). Sometimes more than one spectral order is imaged on the same detector in a so-called cross-dispersed configuration. It is also possible to place a number of slits next to each other and redirect the resulting spectra to different locations on the same detector. Such an instrument is known as a slicer-type IFU, and will produce a line image cube in which each pixel contains a spectrum (a so-called spaxel). Other types of IFUs exist but, for this paper, only a slicer is considered. A slicer IFU data cube can also be constructed using a single-slit instrument by stepping the slit across the source, although this approach obviously results in a great loss in efficiency as compared to an IFU.

The simulated observables are calculated as follows. The basic RADLite output is an intensity map as a function of velocity offset, $v$, from a line center, $I(x, y, v)$, where the spatial coordinates, $(x, y)$, are measured in angular units, assuming some distance to the source. A telescopic observation causes the intensity map to be convolved with an image $\operatorname{PSF}, \operatorname{PSF}(x, y)$, and an instrumental line broadening profile, $L(v)$, resulting in an "telescopic" intensity map:

$$
I_{T}(x, y, v)=[I(x, y, v) * \operatorname{PSF}(x, y)] * L(v),
$$

where $*$ denotes the convolution operator.

The echellogram, as observed by a single-slit grating spectrometer, is simply the telescopic intensity map integrated over the entrance slit width. The slit is oriented at a position angle, P.A., on the sky (negative rotation), with width, $W$, such that the coordinates defined by the slit become, $\xi=x \cos ($ P.A. $)-y \sin ($ P.A.) and $\eta=x \sin ($ P.A. $)+y \cos ($ P.A.). In this system, $\xi$ and $\eta$ are coordinates across and along the slit, 
respectively. The echellogram becomes

$$
E(\eta, v)=\int_{-W / 2}^{W / 2} I_{T}(\eta, \xi, v) d \xi .
$$

The advantage of the echellogram is that it encompasses both spatial and spectral information.

The line profile is a higher level product, being the echellogram integrated over some chosen aperture along the slit $(\eta)$ with width, $A$. Often this integral is weighted by the signal strength in the $\eta$ direction, resulting in a so-called optimal extraction. However, since that approach can be difficult to quantify for extended sources, it is not used here. The line profile is

$$
F(v)=D_{A} \times \int_{-A / 2}^{A / 2} E(\eta, v) d \xi,
$$

where $D_{A}$ is a constant factor, depending on the shape of the PSF, of order unity taking into account that $A$ may not be large enough to contain all the source flux. If there is no noise added to the model, $A$ can be chosen sufficiently large so that $D_{A}=1$. It is also possible to use the echellogram to calculate the astrometric (first moment) spectrum. Following Pontoppidan et al. (2008),

$$
S A(v)=C_{A} \times \frac{\int_{-A / 2}^{A / 2} E(\eta, v) \eta d \eta}{\int_{-A / 2}^{A / 2} E(\eta, v) d \eta},
$$

where $C_{A}$ is a constant factor that approaches unity as $A \rightarrow$ $\infty$, similar to $D_{A}$. This spectrum is used to extract spatial information on scales smaller than the formal spatial resolution of the telescopic image. Note that if there is a strong continuum underlying the line, the astrometric spectrum of the line will be "diluted" by a factor depending on the line-to-continuum ratio: $\left(1+F_{\text {continuum }} / F_{\text {line }}\right)$. In other words, the measured astrometric spectrum as given in Equation (6) should be multiplied by this factor to determine the real, continuum-subtracted, extent of the line emission.

\subsection{PSF Modeling}

In order to estimate the quality of telescopic line images, an idealized diffraction PSF of an ELT is calculated and used to convolve model images. We use the analytical approach of Sabatke et al. (2005) relevant for pupil masks consisting of hexagonal segments. Note that we do not attempt to accurately model an actual telescope, since the various ELTs are still in the design phase. In particular, the influence on the pupil mask by the presence of secondary mounts has been ignored, and the segment alignment is assumed to be perfect. The adopted ELT mirror is inspired by that planned for the European ELT (E-ELT), and consists of 984 hexagonal segments, each $1.4 \mathrm{~m}$ wide, resulting in a $42 \mathrm{~m}$ aperture.

\subsection{Atmospheric Transmission and Correction for Telluric Lines}

A fundamental problem for ground-based infrared spectroscopy is the presence of strong absorption lines from molecules in the Earth's atmosphere (telluric lines). These lines are often the same transitions that may be targeted in astronomical objects, and protoplanetary disks in particular. If the telluric line is saturated, it is necessary to observe the target at a time of the year when its radial velocity is large enough to shift the line away from its telluric counterpart. Some astronomical objects have large intrinsic radial velocities, but most nearby protoplanetary disks do not. Typically, shifts of order $20-30 \mathrm{~km} \mathrm{~s}^{-1}$ are required, which, by a coincidence of nature, are similar to the velocity of the Earth relative to the Sun. To simulate these circumstances, we use a transmission model calculated for Paranal in Chile, the location of the European Very Large Telescope, and assume an optimal, but generally achievable, velocity shift of $30 \mathrm{~km} \mathrm{~s}^{-1}$.

Space-based infrared observatories, such as the James Webb Space Telescope (JWST), obviously do not require the correction for telluric absorption. The Stratospheric Observatory for Infrared Astronomy (SOFIA) has a significant atmospheric column above it for terrestrially abundant species such as $\mathrm{CO}_{2}$, $\mathrm{CH}_{4}$, or $\mathrm{H}_{2} \mathrm{O}$, and hence still requires a correction for the telluric transmission spectrum.

\subsection{Sensitivity}

An important component for predicting telescope performance is the sensitivity of the modeled observation. For a future ELT, predicting detailed sensitivities is clearly a futile task. For illustrative purposes, we use point-source sensitivities of $125 \mu \mathrm{Jy}$ ( $1 \sigma$ in $1 \mathrm{hr})$ at the $M$ band $(4.6 \mu \mathrm{m})$ and $370 \mu \mathrm{Jy}$ at the $N$ band $(10.5 \mu \mathrm{m})$ for resolving powers of 100,000 and 50,000, respectively. These are values calculated using the online exposure time calculator for the E-ELT, available on the ESO Web site. The $M$-band sensitivity is only $\sim 20$ times better than that of VLT-CRIRES, which seems to be a conservative estimate. For instance, on the Web site for METIS, the high-resolution mid-infrared spectrometer E-ELT concept, the point-source sensitivities are reported to be $\sim 10$ times better at $4.6 \mu \mathrm{m}$. At the $N$ band, the gain seems to be significantly higher compared to VLT-VISIR, according to all available estimates. Sensitivities for existing instrumentation are based on actual typical performance.

\section{MOLECULAR LINE IMAGING IN THE INFRARED}

A key property of ground-based telescopes with extremely large apertures is their potential for delivering imaging with very high spatial resolution in the infrared-where the performance of adaptive optics systems is expected to deliver nearly diffraction limited images with a resolution as high as 10-25 mas at $2-5 \mu \mathrm{m}$ and $50-100$ mas at $10-20 \mu \mathrm{m}$. This corresponds to physical scales of 2-10 AU in nearby star-forming regions, which offer up to 1000 potential targets including PP disks and other types of young stellar objects (YSOs) across the stellar mass range from the brown dwarf limit to young A stars (Evans et al. 2009). In this section, we demonstrate how RADLite can be applied to existing molecular line spectra and explore what images of PP disks in various infrared molecular lines are expected to look like with a mid-infrared imaging spectrometer operating with spectral resolving powers of 50,000-100,000 mounted on an ELT. Several concepts for such ELT instruments are currently being developed. While the focus of the RADLite demonstration is on line imaging with ground-based facilities, there are other important facilities for mid-infrared spectroscopy, each covering unique aspects. The mid-infrared spectrometer on the $J W S T$, MIRI, will provide highly sensitive medium resolution spectroscopy across the mid-infrared range (5-27 $\mu \mathrm{m})$. For the brightest sources, the high-resolution spectrometer on SOFIA, EXES, will enable spectrally resolved observations of a wide range of lines, not available from the ground, including the bending mode lines of water around $6 \mu \mathrm{m}$, in addition to other 


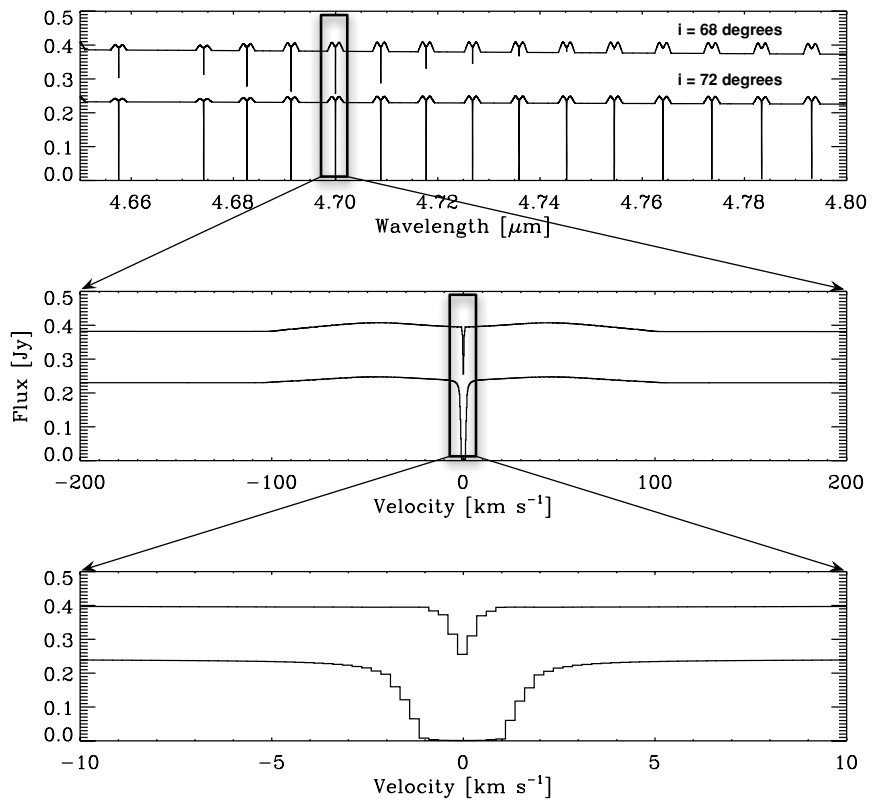

Figure 5. Spectrum of the fundamental ${ }^{12} \mathrm{CO}$ rovibrational band for a disk at inclination angles of $68^{\circ}$ and $72^{\circ}$, as rendered by RADLite, showing both emission and absorption components. The spectral resolving power is assumed to be very high $\left(0.1 \mathrm{~km} \mathrm{~s}^{-1}\right)$. This model illustrates that RADLite can efficiently, and within one instance, deal with spatial scales ranging from $<0.1 \mathrm{AU}$ to $>100 \mathrm{AU}$ as well as spectral scales ranging from $<0.1 \mathrm{~km} \mathrm{~s}^{-1}$ to the entire spectral energy distribution (SED).

important species such as $\mathrm{CH}_{4}$. $\mathrm{RADLite}$ is also intended to aid in the modeling of observations from these facilities.

\subsection{Fiducial Disk Model}

We base most of the examples on the fiducial disk model described in detail in a companion paper (Model 5 of Meijerink et al. 2009), but note the cases where departures from the fiducial model are made for illustrative purposes, such as introducing an inner hole to simulate transition disks. Briefly, the central source is a $M=1 M_{\odot}, R=2 R_{\odot}$ star with an effective temperature of $T=4275 \mathrm{~K}$, corresponding to a luminosity of $1.4 L_{\odot}$ and an age of $2 \mathrm{Myr}$ (Siess et al. 2000). The stellar input spectrum is a model atmosphere from Kurucz (1993). The disk has a gas mass of $10^{-2} M_{\odot}$, an outer radius of $120 \mathrm{AU}$, a Gaussian vertical density profile and a surface density that varies with radius as $\Sigma \sim R^{-1}$. The inner rim is roughly located at the dust sublimation radius corresponding to $1600 \mathrm{~K}$. The disk is assumed to have already experienced some grain growth, simulated by an enhanced gas-to-dust ratio of 12,800 , a low flaring index of $H / R \sim R^{-0.1}$, and an outer scale height $H_{\text {out }} / R_{\text {out }}=0.1$. The total gas-to-solid ratio will of course still be 100; the idea is to model the case where $99 \%$ of the dust has already grown to centimeter or meter sizes, at which point they no longer contribute significantly to the infrared opacity. Further, these large grains will likely have settled to the midplane, and will therefore no longer contribute to the disk surface properties. The local line broadening is assumed to be dominated by the thermal component, corresponding to a low turbulent broadening, assumed to be a constant fraction of the local sound speed: $v_{\text {turb }}=0.03 c_{s}$. The $\mathrm{CO}$ abundance is a constant $2 \times 10^{-4}$ per hydrogen nucleus. The maximal water abundance (gas + ice) is taken to be high, $3 \times 10^{-4}$ per hydrogen nucleus (i.e., $\mathrm{H}+2 \mathrm{H}_{2}$ ) in shielded regions. As discussed in Meijerink et al. (2009), the innermost disk has the maximal water abundance,

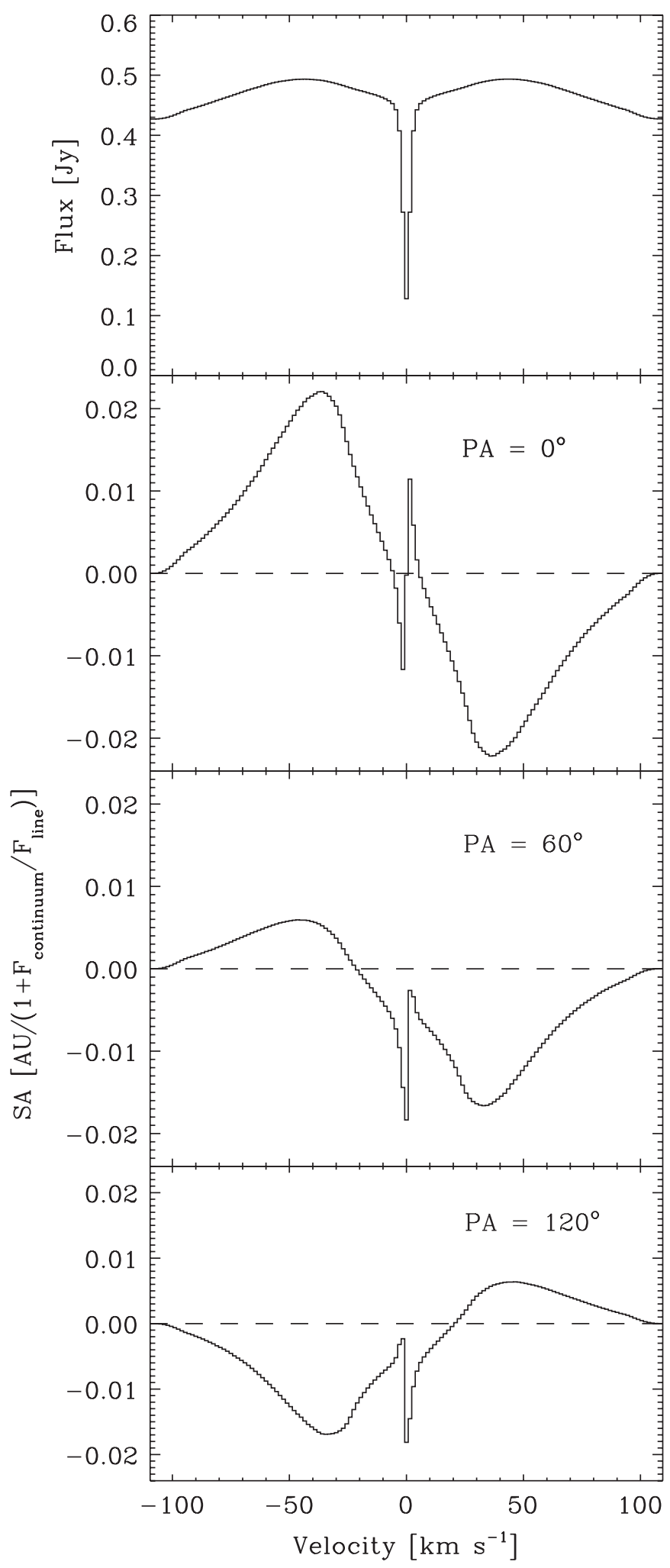

Figure 6. Simulated astrometric spectra $(S A(v)$, Equation (6)) of the CO rovibrational $v=1-0 \mathrm{P}(8)$ line for the fiducial T Tauri star model. The data are simulated for a resolving power of $3 \mathrm{~km} \mathrm{~s}^{-1}$, as is offered by, e.g., CRIRES on the VLT. The top panel shows the flux spectrum for a source at $125 \mathrm{pc}$, while the lower three panels show the astrometric spectra at three different slit P.A.s.

while the surface outward of the mid-plane snow line, located at $0.7 \mathrm{AU}$, is strongly depleted in water down to a fractional abundance of $10^{-10}$ per hydrogen nucleus in order to match 


\section{VLT-CRIRES SR 21 CO P(8)}

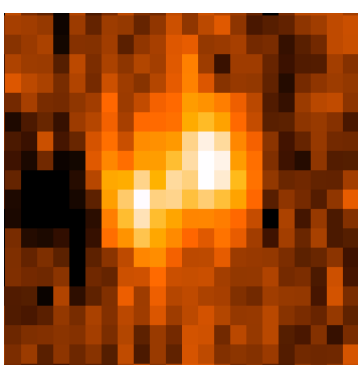

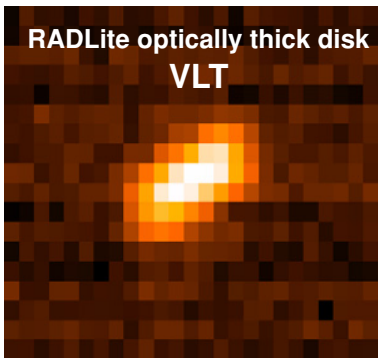

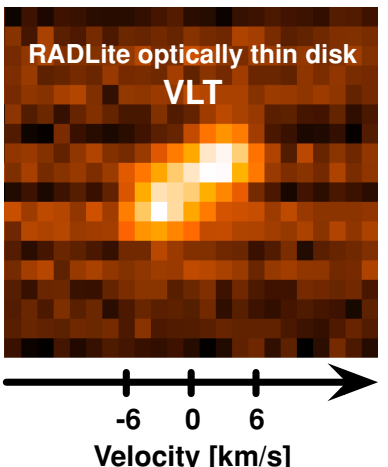

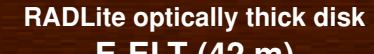

E-ELT (42 m)
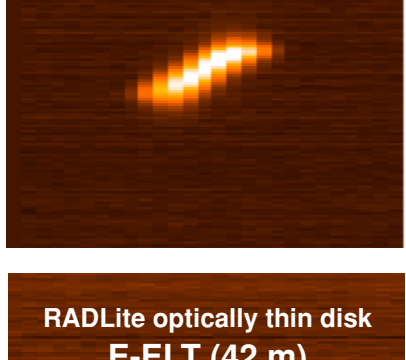

E-ELT (42 m)

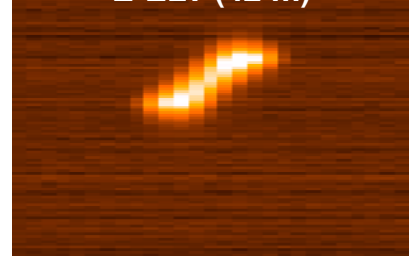

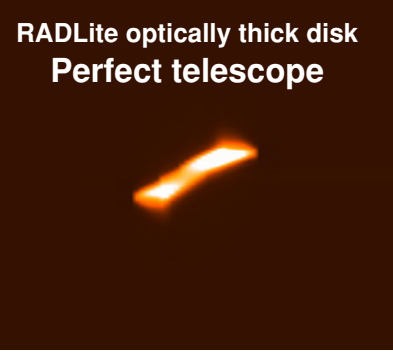

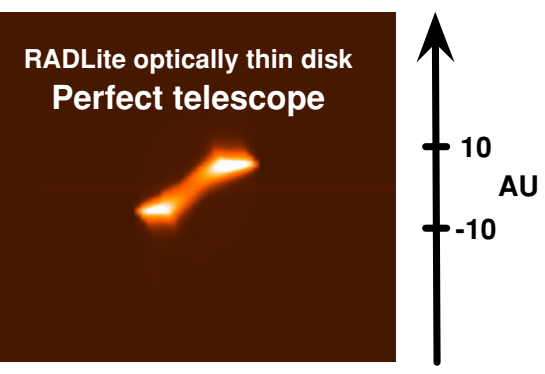

Figure 7. Example of a VLT-CRIRES continuum-subtracted echellogram $(E(\eta, v)$, Equation (4)) of the $\mathrm{P}(8)$ CO line from the $v=1-0$ fundamental band at $4.736 \mu \mathrm{m}$ toward SR 21. The line is marginally resolved in the spatial direction, with a blue-to-red peak offset of $13 \mathrm{AU}$, as determined using spectro-astrometry (Pontoppidan et al. 2008). The right six panels show RADLite models of the line using the disk parameters of Pontoppidan et al. (2008), i.e., a $1.5 M_{\odot}, 6500 \mathrm{~K}, 3 R_{\odot}$ star with a disk with an $R=6.5 \mathrm{AU}$ gap, viewed at an inclination of $20^{\circ}$. Both the observation and model have slits oriented at an angle of $10^{\circ}$ from the disk major axis. Otherwise, the disk is the fiducial model, with a CO abundance of $2 \times 10^{-4}$ per hydrogen nucleus. The top panels is a model that assumes a gas-to-dust ratio of 1280 and is optically thick in the continuum at $4.7 \mu \mathrm{m}$; the lower panels show a disk where the gas-to-dust ratio has been increased to $2.6 \times 10^{5}$, at which point the disk becomes optically thin to continuum photons at $4.7 \mu \mathrm{m}$ (see the discussion in the text). The resolving power is $3 \mathrm{~km} \mathrm{~s}^{-1}$ for both models and observation. The horizontal noise streaks is an artifact of the continuum subtraction due to the noise of the continuum frame being reproduced at each velocity. Hence, the streaks would also appear in real data. Note that the models assume diffraction limited imaging (FWHM $=122$ mas at $4.7 \mu \mathrm{m}$ ), while the actual CRIRES observation probably has a significant seeing halo from incomplete AO correction. The point-source sensitivity for the ELT observation is assumed to be $1.3 \times 10^{-4} \mathrm{Jy} \mathrm{rms} \mathrm{for} \mathrm{a} 1 \mathrm{hr}$ observation, which is about 20 times better than that of VLT-CRIRES.

(A color version of this figure is available in the online journal.)

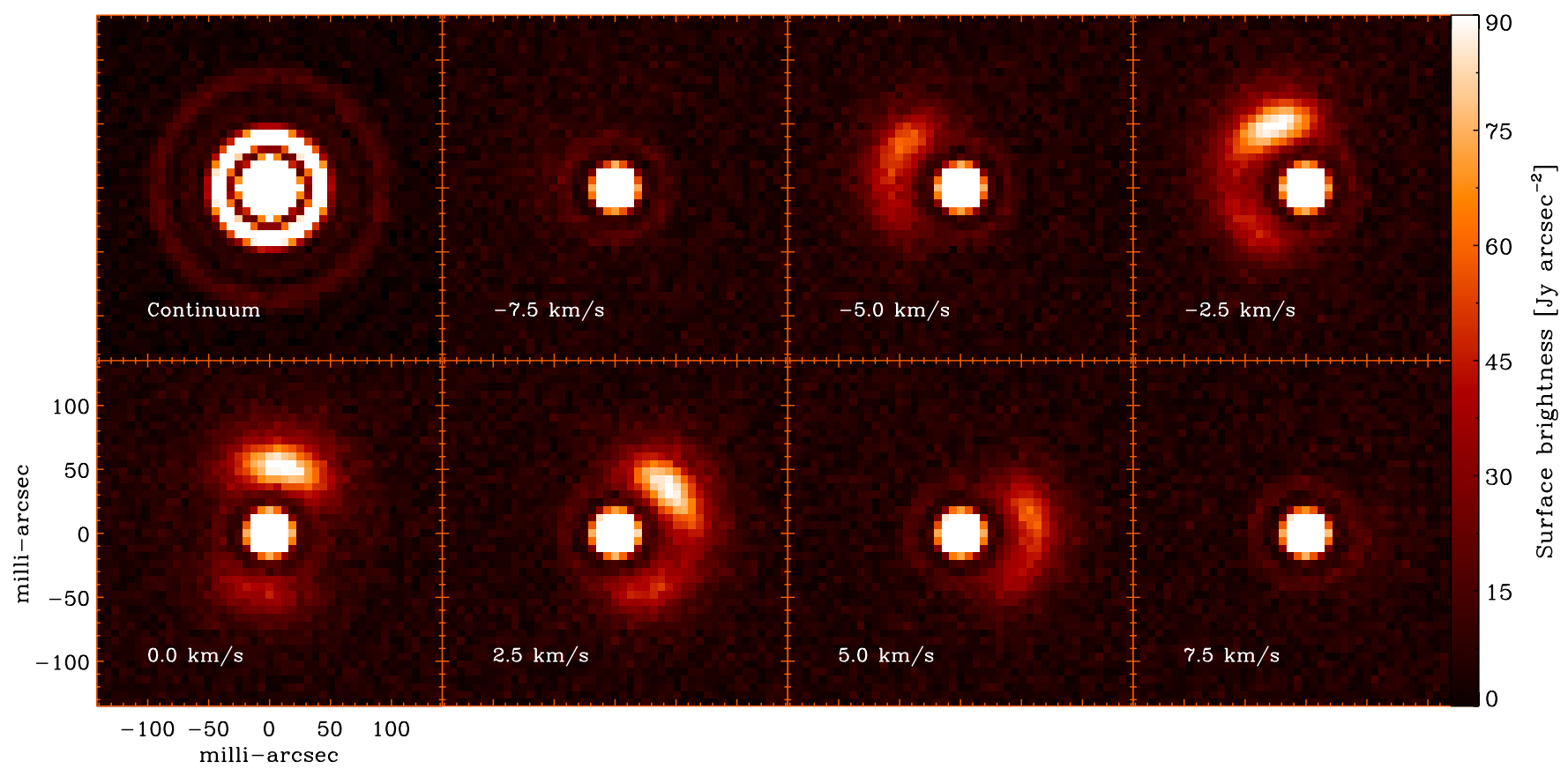

Figure 8. Image cube $\left(I_{T}(x, y, v)\right.$, Equation (3)) of a simulated E-ELT IFU observation of the $v=1-0 \mathrm{P}(8)$ CO line at $4.736 \mu \mathrm{m}$ for SR 21 at an assumed distance of $125 \mathrm{pc}$, including an appropriate noise level, as discussed in the text. The far side of the disk is toward the top. The scale of the image is linear. This simulation can be compared to the simulated single-slit observation in Figure 7. The first panel shows the continuum image obtained bluewards of the line. $90 \%$ of the continuum has been subtracted from the remaining panels to illustrate that the minimum required differential imaging contrast is only 1:10.

(A color version of this figure is available in the online journal.) 
observed mid-infrared water spectra in T Tauri disks. The level populations of the water vapor are calculated in non-LTE using beta3D, while the $\mathrm{CO}$ is assumed to be in LTE. A complete, quantitative description of the RADLite input model, such as the density, temperature, and abundance distributions, as well as a detailed treatment of the excitation of water vapor, can be found in Meijerink et al. (2009).

\subsection{The CO Fundamental}

Given the large volume of observations of the $\mathrm{CO}$ fundamental, it is expected that modeling of these lines will be a central application of RADLite. CO rovibrational lines are ubiquitous tracers of disk surfaces at $\sim 1$ AU (Najita et al. 2003; Blake $\&$ Boogert 2004; Brittain et al. 2007; Salyk et al. 2007). Some disks are already being resolved in $\mathrm{CO}$ rovibrational lines with adaptive optics (AO)-fed spectrometers on $8-10 \mathrm{~m}$ class telescopes (Goto et al. 2006). With the improvement in spatial resolution of an ELT by factors of 3-5, the CO emission from many disks should be imaged over a correspondingly larger number of spaxels. Further, the $\mathrm{CO}$ fundamental can be used to demonstrate the ability of the code to simultaneously treat dynamic ranges of 4-5 orders of magnitude in both velocity and spatial dimension. Figure 5 shows a rendering of the ${ }^{12} \mathrm{CO}$ band (lines from $\mathrm{R}(0)$ to $\mathrm{P}(14)$ ) for the fiducial disk model viewed at high inclination $\left(\sim 70^{\circ}\right)$. The models exhibit double-peaked emission line profiles typical for Keplerian disks. The lines are formed in the inner $1 \mathrm{AU}$ of the disk. Superposed on the emission lines are very narrow absorption lines formed as continuum and line emission from the inner disk are absorbed by the outer disk - a configuration made possible by a flaring disk geometry. Such inclined disks have been used in the past to study disk material through absorption features (Pontoppidan et al. 2005; Brittain et al. 2005; Lahuis et al. 2006; Gibb et al. 2007). Two different models are produced, for inclinations of $68^{\circ}$ and $72^{\circ}$, illustrating how rapidly the $\mathrm{CO}$ absorption changes with inclination; essentially no absorption is seen at inclinations below $68^{\circ}$, while the central continuum becomes very faint $(\ll 0.1 \mathrm{Jy})$ at inclinations higher than $75^{\circ}$. The prediction is that only $\sim 5 \%$ of disks will have the appropriate inclination to show absorption from the outer disk. Qualitatively, the model spectrum at $72^{\circ}$ inclination resembles that of HL Tau, a disk suggested to have a large gas-to-dust ratio in its upper layers, possibly due to dust settling (Brittain et al. 2005).

There are currently no high-resolution IFU spectrometers that operate in the 3-20 $\mu \mathrm{m}$ region. However, one imaging technique that has been used for $\mathrm{CO}$ is spectro-astrometry (Pontoppidan et al. 2008; van der Plas et al. 2009). Figure 6 shows astrometric spectra at three different P.A.s of the $i=72^{\circ} \mathrm{P}(8) \mathrm{CO}$ rovibrational line also seen in Figure 5. At a P.A. $=0^{\circ}$, the classical antisymmetric profile of a Keplerian disk appears. The absorption from the outer disk shows up in the astrometry as a second antisymmetric profile - but with the opposite sign of that produced by the emission. This signature is due to the Keplerian rotation of the outer disk having a significantly varying radial component across the emission source (which consists of the central star, thermal emission from the innermost part of the disk, as well as scattering from the disk surface), and can therefore potentially be used to distinguish $\mathrm{CO}$ absorption from the disk, as opposed to $\mathrm{CO}$ absorption from an unrelated foreground cloud (which would have no astrometric signature). Also notable is a significant asymmetry seen in the off-axis angles 60 and $120^{\circ}$. For instance, at $60^{\circ}$, the blueshifted lobe has a much smaller offset than the redshifted lobe. This asymmetry is due to the
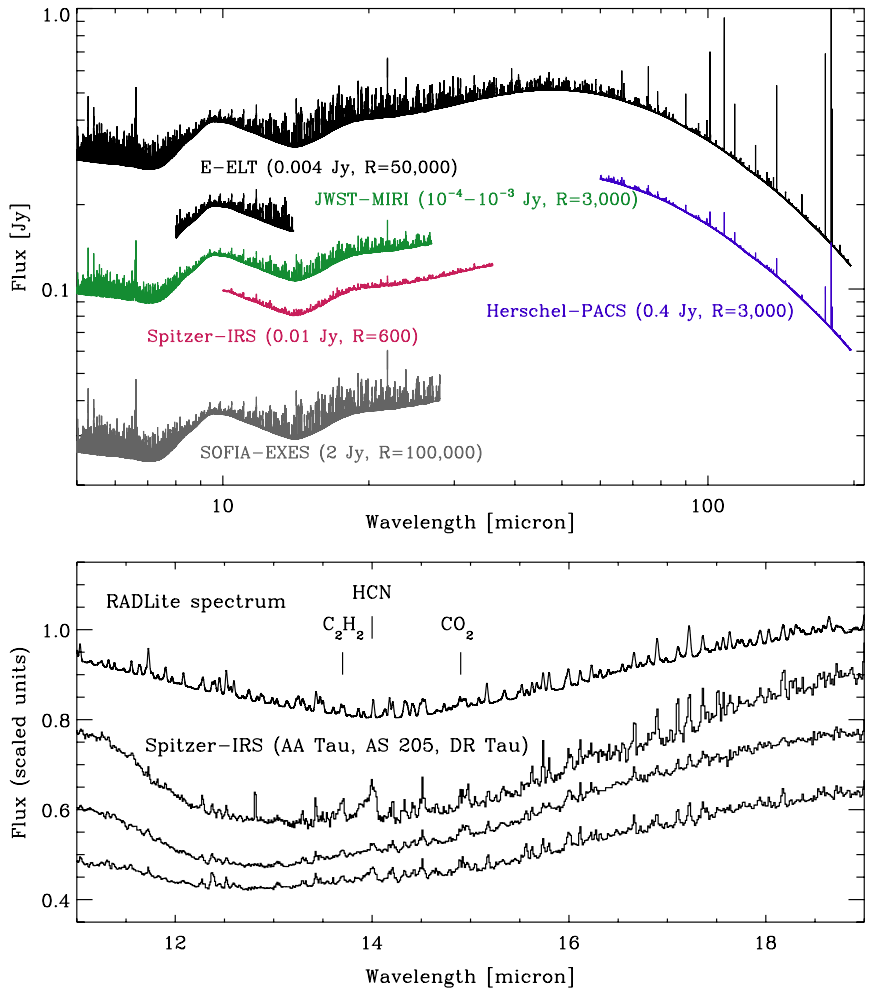

Figure 9. Top panel: a full range calculation of the infrared water spectrum from a typical protoplanetary disk (Model 5 in Meijerink et al. 2009). In addition, scaled (for clarity) model spectra convolved to the spectral resolution of various infrared observatories are shown. For each observatory and instrument, rough sensitivities $(\sim 10 \sigma$ in $1 \mathrm{hr})$ and spectral resolving powers are indicated. Bottom panel: a comparison of the model convolved to the Spitzer resolution (top curve) with Spitzer-IRS spectra of typical protoplanetary disks around solar-mass stars (from top to bottom, AA Tau, AS 205 and DR Tau, scaled for clarity, from Carr \& Najita 2008; Salyk et al. 2008). Note that the model only includes water lines, not the $Q$-branches from $\mathrm{C}_{2} \mathrm{H}_{2}, \mathrm{HCN}$, and $\mathrm{CO}_{2}$, which are prominent in the data. The observed spectra show lines of many more molecular and atomic species.

combination of two effects. First, the structure of the inner rim in the disk causes an asymmetry of the continuum. When viewed at an inclined angle, the photocenter of the continuum is shifted in the direction of the far side of the disk. Because spectroastrometry is a relative measurement of a line compared to a continuum, this effect takes the appearance of a line asymmetry. Second, there is a true line asymmetry due to the fact that the disk is not perfectly flat. Thus, the surface isovelocity contours are not symmetric around the disk major axis, and the angle of a ray with respect to the disk surface normal is larger from the near side of the disk relative to the far side. The asymmetries are strong enough to be easily detectable with current spectro-astrometric facilities such as CRIRES on the Very Large Telescope (VLT), and can be effectively modeled with RADLite.

The large apertures of the ELTs will be able to produce line images of planet-forming regions across many spaxels. The fundamental rovibrational band of $\mathrm{CO}$ will be the easiest imaging target. The expected improvement of a $42 \mathrm{~m}$ telescope relative to an $8.2 \mathrm{~m}$ telescope is illustrated in Figures 7 and 8 . The former shows an actual single-slit observation of a $\mathrm{CO}$ line from the protoplanetary disk SR 21 (see Pontoppidan et al. 2008, for details), which has an evolved planet-forming region characterized by a large inner hole (a transitional or cold disk). The observation is modeled with RADLite using the basic disk geometry derived by Pontoppidan et al. (2008), who 


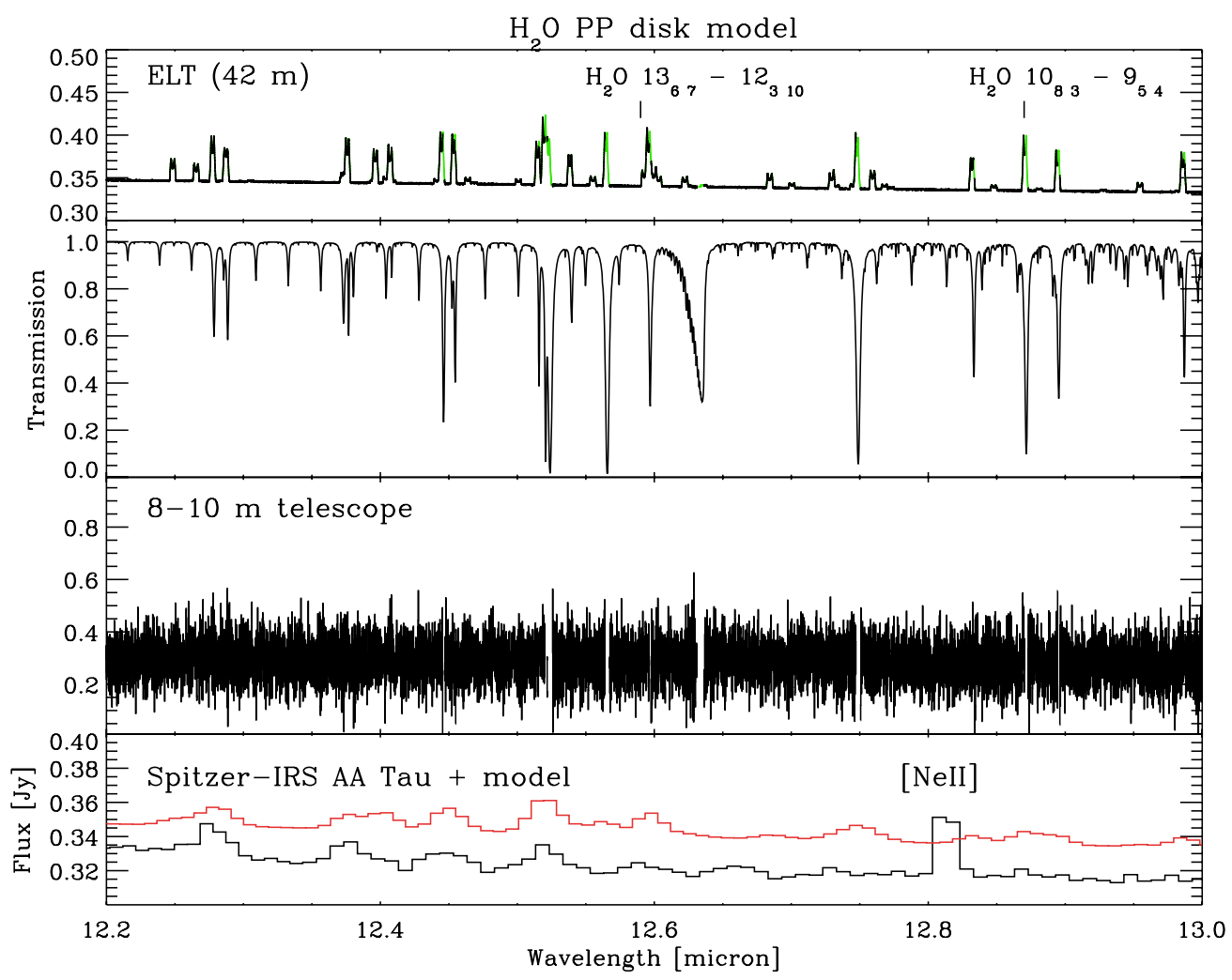

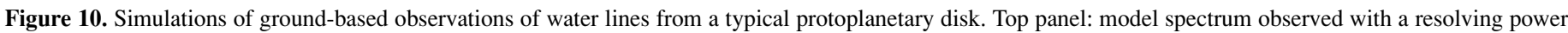

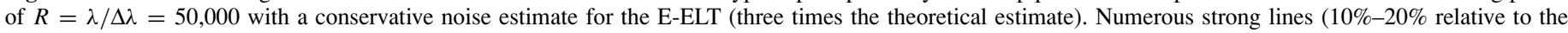

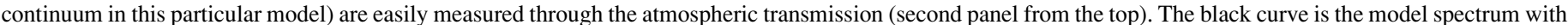

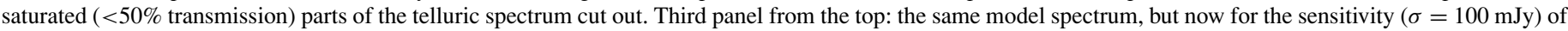

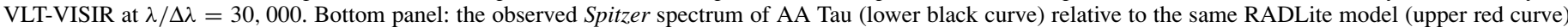

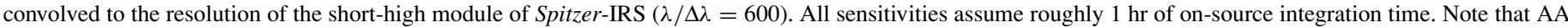
Tau is not a particularly bright target; with a continuum flux of $0.3 \mathrm{Jy}$ at $10 \mu \mathrm{m}$, hundreds of protoplanetary disks will be brighter, some by an order of magnitude.

determined that the $\mathrm{CO}$ gas is truncated within 6.5 AU. The additional applicability of RADLite is illustrated by simulating observations using two different gas-to-dust ratios to simulate the effect of grain growth. The low gas-to-dust ratio is 1280 while the high gas-to-dust ratio of $2.56 \times 10^{5}$ has lowered the disk opacity sufficiently to make the disk optically thin to the continuum emission at $4.7 \mu \mathrm{m}$. This produces two emission lobes in the position-velocity image, in contrast to the optically thick disk, which results in a single elongated structure. This difference is due to a limb brightening effect of the inner rim of the disk. For an optically thick inner rim, the surface intensity of the inner wall is constant at different ray impact parameters; the inner rim acts as an opaque "wall." An optically thin disk will result in a higher intensity at image locations where a ray is near tangential to the inner rim, i.e., where a ray samples the largest column density of warm gas. While the observation hints at an optically thin disk, the greater sensitivity and higher spatial resolution of an ELT observation will enable such conclusions to be made with much greater confidence and for a much wider range of disks.

It is likely that an ELT mid-infrared instrument will not be a single-slit facility, but an IFU. Figure 8 shows a RADLite simulation of the same disk as observed with a $42 \mathrm{~m}$ aperture telescope equipped with an IFU. In this case, the $\mathrm{CO}$ emission would be imaged across at least 50 spaxels within $12 \mathrm{AU}$. It will thus be possible to search for kinematic structures in the disk directly related to planet formation, such as spiral waves and the radial density structure in the optically thin parts of the disk.
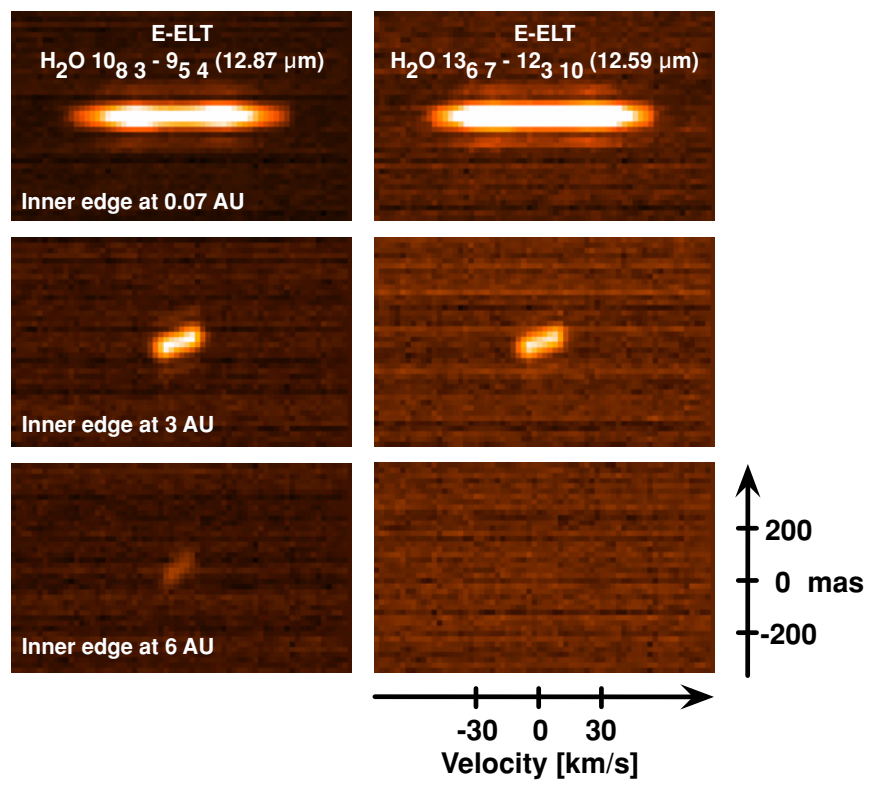

Figure 11. Simulated E-ELT continuum-subtracted echellograms of two $N$-band $\mathrm{H}_{2} \mathrm{O}$ lines for the fiducial non-LTE $\mathrm{H}_{2} \mathrm{O}$ model, both of which require velocity shifts of $\sim 30 \mathrm{~km} \mathrm{~s}^{-1}$ to shift the lines out of the telluric absorption line. The left line has an upper level energy of $3243 \mathrm{~K}$; the right has an upper level energy of $3966 \mathrm{~K}$. In the lower two rows of panels, models with a $3 \mathrm{AU}$ and $6 \mathrm{AU}$ inner hole are also calculated. For clarity, the atmospheric transmission is not simulated.

(A color version of this figure is available in the online journal.) 
Table 1

Strong $\mathrm{N}$-band $\mathrm{H}_{2} \mathrm{O}$ Lines Suitable for Ground-based Observations

\begin{tabular}{|c|c|c|c|c|c|c|c|}
\hline \multicolumn{3}{|c|}{ Transition $^{\mathrm{a}}$} & \multirow{2}{*}{$\begin{array}{c}\text { Wavelength }(\mu \mathrm{m})^{\mathrm{b}} \\
8.09583\end{array}$} & \multirow{2}{*}{$\begin{array}{c}\text { Frequency }\left(\mathrm{cm}^{-1}\right) \\
1235.20415\end{array}$} & \multirow{2}{*}{$\frac{{\text { Spitzer } \text { Detected }^{\mathrm{c}}}_{\text {N/A }}}{\text { N }}$} & \multirow{2}{*}{$\begin{array}{c}\text { Strength }^{\mathrm{d}} \\
0.09\end{array}$} & \multirow{2}{*}{$\begin{array}{c}\text { Transmission } \\
\text { Clean }\end{array}$} \\
\hline o $v=1-0$ & $17_{017}$ & $\rightarrow \quad 18_{118}$ & & & & & \\
\hline o $v=1-0$ & $11_{83}$ & $\rightarrow \quad 12_{94}$ & 8.10842 & 1233.28658 & N/A & 0.09 & Clean \\
\hline o $v=1-0$ & 1294 & $\rightarrow 13_{103}$ & 8.19373 & 1220.44594 & N/A & 0.09 & Clean \\
\hline o $v=1-0$ & $11_{65}$ & $\rightarrow 12_{76}$ & 8.19441 & 1220.34459 & $\mathrm{~N} / \mathrm{A}$ & 0.05 & Clean \\
\hline $\mathrm{p} v=1-0$ & $11_{66}$ & $\rightarrow 12_{75}$ & 8.20245 & 1219.14720 & N/A & 0.05 & Clean \\
\hline $\mathrm{p} v=1-0$ & $18_{018}$ & $\rightarrow \quad 19_{119}$ & 8.23469 & 1214.37420 & $\mathrm{~N} / \mathrm{A}$ & 0.08 & Clean \\
\hline $\mathrm{p} v=1-0$ & $11_{57}$ & $\rightarrow 12_{66}$ & 8.24397 & 1213.00716 & N/A & 0.07 & Clean \\
\hline o $v=1-0$ & $12_{85}$ & $\rightarrow 13_{94}$ & 8.26608 & 1209.76296 & N/A & 0.06 & Clean \\
\hline o $v=1-0$ & $12_{76}$ & $\rightarrow 13_{85}$ & 8.32319 & 1201.46258 & $\mathrm{~N} / \mathrm{A}$ & 0.05 & Clean \\
\hline o $v=0-0$ & 1367 & $\rightarrow \quad 12_{112}$ & 8.34329 & 1198.56748 & N/A & 0.05 & Clean \\
\hline o $v=1-0$ & $12_{67}$ & $\rightarrow 13_{76}$ & 8.36579 & 1195.34395 & $\mathrm{~N} / \mathrm{A}$ & 0.06 & Clean \\
\hline o $v=1-0$ & $13_{85}$ & $\rightarrow 14_{96}$ & 8.42794 & 1186.52920 & N/A & 0.05 & Clean \\
\hline o $v=1-0$ & 1258 & $\rightarrow 13_{67}$ & 8.44520 & 1184.10422 & $\mathrm{~N} / \mathrm{A}$ & 0.06 & Clean \\
\hline o $v=1-0$ & 1249 & $\rightarrow 13_{58}$ & 8.76006 & 1141.54447 & $\mathrm{~N} / \mathrm{A}$ & 0.05 & Clean \\
\hline o $v=1-0$ & $9_{27}$ & $\rightarrow 10_{56}$ & 9.09356 & 1099.67926 & N/A & 0.06 & Clean \\
\hline o $v=1-0$ & $10_{29}$ & $\rightarrow 11_{38}$ & 9.16418 & 1091.20494 & N/A & 0.06 & Clean \\
\hline o $v=0-0$ & $14_{69}$ & $\rightarrow 13_{12}$ & 9.59209 & 1042.52515 & $\mathrm{~N} / \mathrm{A}$ & 0.05 & Clean \\
\hline o $v=0-0$ & 19217 & $\rightarrow \quad 18_{118}$ & 9.88515 & 1011.61860 & $\mathrm{~N} / \mathrm{A}$ & 0.09 & Clean \\
\hline o $v=0-0$ & $14_{105}$ & $\rightarrow 1376$ & 10.05642 & 994.38945 & No & 0.05 & Clean \\
\hline o $v=0-0$ & 17710 & $\rightarrow \quad 16_{413}$ & 10.11319 & 988.80730 & No & 0.05 & Clean \\
\hline o $v=0-0$ & $17_{89}$ & $\rightarrow 16_{512}$ & 10.27482 & 973.25273 & No & 0.05 & Clean \\
\hline o $v=0-0$ & $13_{103}$ & $\rightarrow 1276$ & 10.30317 & 970.57472 & No & 0.05 & Clean \\
\hline o $v=0-0$ & 18316 & $\rightarrow \quad 17_{117}$ & 10.42505 & 959.22783 & No & 0.10 & Clean \\
\hline o $v=0-0$ & 1698 & $\rightarrow 15_{69}$ & 10.47955 & 954.23969 & No & 0.05 & Clean \\
\hline o $v=0-0$ & 1258 & $\rightarrow 11_{011}$ & 10.54560 & 948.26294 & No & 0.06 & Clean \\
\hline o $v=0-0$ & $14_{96}$ & $\rightarrow 13_{67}$ & 10.76434 & 928.99316 & No & 0.07 & Clean \\
\hline o $v=0-0$ & 1276 & $\rightarrow 11_{29}$ & 10.84439 & 922.13542 & No & 0.08 & Clean \\
\hline o $v=0-0$ & $15_{69}$ & $\rightarrow \quad 14_{312}$ & 10.85308 & 921.39780 & No & 0.09 & Clean \\
\hline o $v=0-0$ & 1394 & $\rightarrow 12_{67}$ & 10.94112 & 913.98290 & No & 0.08 & Clean \\
\hline o $v=0-0$ & 19415 & $\rightarrow 18_{316}$ & 10.97414 & 911.23324 & No & 0.07 & Clean \\
\hline o $v=0-0$ & $18_{415}$ & $\rightarrow 17_{116}$ & 10.98045 & 910.70960 & No & 0.09 & Clean \\
\hline o $v=0-0$ & $12_{67}$ & $\rightarrow 11_{110}$ & 11.00168 & 908.95248 & No & 0.10 & $>30 \mathrm{~km} \mathrm{~s}^{-1}$ \\
\hline o $v=0-0$ & $15_{87}$ & $\rightarrow \quad 14_{510}$ & 11.02836 & 906.75277 & No & 0.10 & $>30 \mathrm{~km} \mathrm{~s}^{-1}$ \\
\hline $\mathrm{p} v=0-0$ & 17315 & $\rightarrow 16_{016}$ & 11.03376 & 906.30900 & No & 0.05 & Clean \\
\hline o $v=0-0$ & $17_{215}$ & $\rightarrow 16_{116}$ & 11.03475 & 906.22805 & No & 0.10 & Clean \\
\hline $\mathrm{p} v=0-0$ & 1293 & $\rightarrow 11_{66}$ & 11.23479 & 890.09255 & No & 0.05 & Clean \\
\hline o $v=0-0$ & 1294 & $\rightarrow 11_{65}$ & 11.25311 & 888.64354 & No & 0.10 & Clean \\
\hline o $v=0-0$ & 1578 & $\rightarrow \quad 14_{411}$ & 11.32409 & 883.07302 & No & 0.10 & Clean \\
\hline $\mathrm{p} v=0-0$ & $11_{57}$ & $\rightarrow 10_{010}$ & 11.47773 & 871.25270 & No & 0.05 & Clean \\
\hline o $v=0-0$ & $11_{92}$ & $\rightarrow 10_{65}$ & 11.55471 & 865.44774 & No & 0.10 & $>30 \mathrm{~km} \mathrm{~s}^{-1}$ \\
\hline $\mathrm{p} v=0-0$ & $11_{93}$ & $\rightarrow 10_{64}$ & 11.56124 & 864.95886 & No & 0.05 & $>30 \mathrm{~km} \mathrm{~s}^{-1}$ \\
\hline o $v=0-0$ & 17314 & $\rightarrow 16_{215}$ & 11.64764 & 858.54294 & No & 0.12 & Clean \\
\hline $\mathrm{p} v=0-0$ & $14_{68}$ & $\rightarrow 14_{68}$ & 11.71816 & 853.37648 & No & 0.08 & Clean \\
\hline o $v=0-0$ & 16314 & $\rightarrow \quad 15_{015}$ & 11.72455 & 852.91119 & Yes & 0.15 & $>30 \mathrm{~km} \mathrm{~s}^{-1}$ \\
\hline o $v=0-0$ & $16_{89}$ & $\rightarrow \quad 15_{510}$ & 11.82409 & 845.73138 & No & 0.07 & Clean \\
\hline $\mathrm{p} v=0-0$ & $15_{88}$ & $\rightarrow 14_{59}$ & 11.88916 & 841.10204 & No & 0.06 & Clean \\
\hline $\mathrm{p} v=0-0$ & $14_{77}$ & $\rightarrow 13_{410}$ & 11.90026 & 840.31789 & No & 0.09 & Clean \\
\hline o $v=0-0$ & $14_{87}$ & $\rightarrow 13_{58}$ & 11.96812 & 835.55295 & No & 0.10 & Clean \\
\hline $\mathrm{p} v=0-0$ & $13_{86}$ & $\rightarrow 12_{57}$ & 12.09017 & 827.11790 & No & 0.08 & Clean \\
\hline $\mathrm{p} v=0-0$ & $17_{513}$ & $\rightarrow 16_{214}$ & 12.24814 & 816.45022 & Yes & 0.07 & Clean \\
\hline o $v=0-0$ & $12_{85}$ & $\rightarrow 11_{56}$ & 12.27721 & 814.51703 & Yes & 0.15 & $>30 \mathrm{~km} \mathrm{~s}^{-1}$ \\
\hline o $v=0-0$ & 16413 & $\rightarrow 15_{114}$ & 12.37566 & 808.03788 & Yes & 0.15 & $>30 \mathrm{~km} \mathrm{~s}^{-1}$ \\
\hline o $v=0-0$ & 17413 & $\rightarrow 16_{314}$ & 12.39625 & 806.69564 & Yes & 0.12 & Clean \\
\hline $\mathrm{p} v=0-0$ & $16_{313}$ & $\rightarrow \quad 15_{214}$ & 12.40705 & 805.99363 & Yes & 0.13 & Clean \\
\hline o $v=0-0$ & $11_{83}$ & $\rightarrow 10_{56}$ & 12.44483 & 803.54638 & Yes & 0.17 & $>30 \mathrm{~km} \mathrm{~s}^{-1}$ \\
\hline o $v=0-0$ & 1376 & $\rightarrow 12_{49}$ & 12.45346 & 802.98996 & Yes & 0.17 & $>30 \mathrm{~km} \mathrm{~s}^{-1}$ \\
\hline $\mathrm{p} v=0-0$ & $15_{313}$ & $\rightarrow \quad 14_{014}$ & 12.51462 & 799.06551 & Yes & 0.15 & $>30 \mathrm{~km} \mathrm{~s}^{-1}$ \\
\hline $\mathrm{p} v=0-0$ & $11_{84}$ & $\rightarrow 10_{55}$ & 12.53831 & 797.55588 & Yes & 0.10 & Clean \\
\hline o $v=0-0$ & 1367 & $\rightarrow 12_{310}$ & 12.59591 & 793.90838 & Yes & 0.20 & $>30 \mathrm{~km} \mathrm{~s}^{-1}$ \\
\hline
\end{tabular}


Table 1

(Continued)

\begin{tabular}{|c|c|c|c|c|c|c|c|}
\hline \multicolumn{3}{|c|}{ Transition $^{\mathrm{a}}$} & \multirow{2}{*}{$\frac{\text { Wavelength }(\mu \mathrm{m})^{\mathrm{b}}}{12.83197}$} & \multirow{2}{*}{$\begin{array}{c}\text { Frequency }\left(\mathrm{cm}^{-1}\right) \\
779.30366\end{array}$} & \multirow{2}{*}{$\frac{{\text { Spitzer } \text { Detected }^{\mathrm{c}}}_{\text {No }}}{\text { Sol }}$} & \multirow{2}{*}{$\frac{\text { Strength }^{\mathrm{d}}}{0.12}$} & \multirow{2}{*}{$\frac{\text { Transmission }^{\mathrm{e}}}{>30 \mathrm{~km} \mathrm{~s}^{-1}}$} \\
\hline $\mathrm{p} v=0-0$ & $10_{82}$ & $\rightarrow 9_{55}$ & & & & & \\
\hline $\mathrm{p} v=0-0$ & 1257 & $\rightarrow 11_{210}$ & 12.89409 & 775.54902 & No & 0.15 & $>30 \mathrm{~km} \mathrm{~s}^{-1}$ \\
\hline $\mathrm{p} v=0-0$ & $12_{75}$ & $\rightarrow 11_{48}$ & 12.98575 & 770.07497 & No & 0.15 & $>30 \mathrm{~km} \mathrm{~s}^{-1}$ \\
\hline o $v=0-0$ & $16_{512}$ & $\rightarrow 15_{213}$ & 13.03333 & 767.26380 & Yes & 0.12 & Clean \\
\hline o $v=0-0$ & $16_{710}$ & $\rightarrow 15_{411}$ & 13.13245 & 761.47255 & Yes & 0.09 & Clean \\
\hline o $v=0-0$ & $15_{312}$ & $\rightarrow \quad 14_{213}$ & 13.29319 & 752.26468 & Yes & 0.20 & $>30 \mathrm{~km} \mathrm{~s}^{-1}$ \\
\hline
\end{tabular}

Notes.

a Bending mode vibrational quantum number.

b Line parameters from HITRAN2004 (Rothman et al. 2005).

c Subjective estimate limited by severe line blending.

d Peak line flux/continuum flux.

e Is a significant Doppler shift required to observe the transition?

\section{3. $\mathrm{H}_{2} \mathrm{O}$ Lines in the Atmospheric $\mathrm{N}$-band Window}

An important new window for high-resolution spectroscopy that will be opened by ELT class telescopes is the 7.5-13.9 $\mu \mathrm{m}$ atmospheric $N$ band, as well as the $17-25 \mu \mathrm{m}$ atmospheric $Q$ band. The only reason that these windows are currently not very productive for ground-based high-resolution spectroscopic work is that the sensitivity of instruments on 8-10 m class telescopes is not sufficient to reach the vast majority of protoplanetary disks, in particular disks around T Tauri stars now known to exhibit the richest molecular emission spectra (see also recent work by Knez et al. 2009 and Najita et al. 2009 for discussions of the power and limitations of current high-resolution $N$ - and $Q$-band spectroscopy). The massive improvements in sensitivity offered by an ELT will put hundreds or thousands of protoplanetary disks within reach for high-resolution imaging spectroscopy in the $N$ and $Q$ bands.

There are many atomic and molecular species with strong transitions in the $N$ and $Q$ bands. One that has received considerable recent attention is the [Ne II] $12.8 \mu \mathrm{m}$ line (Pascucci et al. 2007; van Boekel et al. 2009; Najita et al. 2009). Here, we show that a large number of rotational lines of water can be reached from the ground. These are lines that are known to be strong in many protoplanetary disks (Carr \& Najita 2008; Salyk et al. 2008, and work in preparation by these authors).

The strong lines and the associated high apparent molecular abundances in the disk surfaces within a few AU were not anticipated by the initial chemical models $-\mathrm{H}_{2} \mathrm{O}$ abundances in the warm surface layers appear to be of order $n_{\mathrm{H}_{2} \mathrm{O}} / n_{\mathrm{H}} \sim$ $10^{-5}-10^{-4}$, while many contemporary models (Glassgold et al. 2004; Gorti \& Hollenbach 2008) predicted significantly less water- $n_{\mathrm{H}_{2} \mathrm{O}} / n_{\mathrm{H}} \sim 10^{-8}-10^{-6}$. Other modelers are now working on explaining very high molecular abundances, including water (Glassgold et al. 2009; Woods \& Willacy 2009).

Strong lines can be found across the entire 10-36 $\mu \mathrm{m}$ Spitzer high-resolution spectral range and, by extension, many strong lines are expected in the $6 \mu \mathrm{m}$ water bending mode as well as in the 40-200 $\mu \mathrm{m}$ range, in the regime of future space-borne observatories. Herschel-PACS will cover lines between 60 and $210 \mu \mathrm{m}$, but will not spectrally resolve them. The first facilities with the potential to produce spatially and spectrally resolved data of rotational water lines from a significant sample of disks will be the ELTs. Figure 9 shows an overview of water lines along with near-term and future infrared facilities. The most sensitive ground-based window for observing the lines is the atmospheric $N$-band window, which extends from $7.5 \mu \mathrm{m}$ to

$13.9 \mu \mathrm{m}$. Figure 10 shows a RADLite model spectrum of the fiducial model, based on observed Spitzer spectra, of part of the $N$ band. The model includes atmospheric absorption for a dry site at $2600 \mathrm{~m}$ elevation. It also assumes that regions of the spectrum with less than $50 \%$ transmission cannot be calibrated, and these parts of the spectrum have been removed. As with most molecular infrared spectroscopy from the ground, it is necessary to observe the target at a time when the Doppler shift due to the relative motion of the Earth is large in order to move the target line away from its telluric counterpart. In this instance, a typical shift of $30 \mathrm{~km} \mathrm{~s}^{-1}$ has been assumed. Finally, noise relevant for current mid-infrared high-resolution spectrometers as well as for an instrument mounted on an ELT has been added to the spectra. It can be seen that for this particular model, approximately $50 \mathrm{H}_{2} \mathrm{O}$ lines will be observable from the ground under dry conditions (1-3 $\mathrm{mm}$ of precipitable water vapor). Most line profiles can be measured in their entirety in one observing epoch, while a few of the lower excitation lines will require the combination of two epochs with opposite Doppler velocity shifts, i.e., separated by six months. The improvement in sensitivity of an ELT as compared to existing ground-based facilities clearly moves this type of observation from being very difficult to being straightforward for a large sample of disks, given that the model example is of typical brightness for nearby T Tauri stars $(0.35 \mathrm{Jy}$ at $10 \mu \mathrm{m})$.

Figure 11 shows simulations of $\mathrm{H}_{2} \mathrm{O}$ line imaging with the E-ELT, assuming a single-slit instrument. The figure shows three different versions of the fiducial non-LTE model: the first with the inner disk truncated at a dust sublimation radius of $0.07 \mathrm{AU}$, the other two assuming inner holes with radii of 3 and $6 \mathrm{AU}$. The echellograms show that a $3 \mathrm{AU}$ hole is easily spatially resolved. Two rotational lines are modeled: $10_{83} \rightarrow 9_{54}$ and $13_{67} \rightarrow 12_{310}$, with upper level excitation energies of $3243 \mathrm{~K}$ and $3966 \mathrm{~K}$, respectively (for comparison, the excitation energy of the $\mathrm{CO}$ rovibrational fundamental is $\sim 3100 \mathrm{~K})$. The low excitation line can be detected out to $6 \mathrm{AU}$, while the higher excitation line drops below the detection limit between 3 and $6 \mathrm{AU}$. Astrometric spectra also detect the offsets for the $0.07 \mathrm{AU}$ truncation radius. This shows that some lines are better tracers of certain radii than others due to a balance between excitation temperatures and geometric enhancement, i.e., the higher excitation line is the brightest at small radii but the faintest at large radii.

In Table 1, an incomplete list of molecular lines most suitable for observations from the ground is given. The list is compiled based on line brightness (more than 5\% relative to 
the continuum) and atmospheric transparency (more than $50 \%$ transmission over at least half the line, assuming $30 \mathrm{~km} \mathrm{~s}^{-1}$ velocity shift relative to the atmosphere). Given that this selection is based on a model of one specific source, in practice, potentially thousands of molecular lines in 3-28 $\mu \mathrm{m}$ range will be detectable from the ground with an ELT equipped with a high-resolution spectrometer.

\section{CONCLUSIONS}

We have presented a new axisymmetric raytracer, called RADLite, for simulating telescopic line images, with specific emphasis on infrared lines formed in the planet-forming region of protoplanetary disks, and applied it to mid-infrared $\mathrm{CO}$ and $\mathrm{H}_{2} \mathrm{O}$ emission. RADLite is optimized for rapidly rendering large numbers of lines for full two-dimensional axisymmetric structures with arbitrarily large velocity gradients, including a detailed treatment of the dust radiative transfer. For instance, a full spectrum of $\sim 1000$ water lines in the infrared $(2-200 \mu \mathrm{m})$ can be rendered with a velocity resolution of $1 \mathrm{~km} \mathrm{~s}^{-1}$ in $1-2 \mathrm{hr}$ on a single workstation. The code has very general applications to chemical and excitation disk models as well as observations from infrared spectrometers on 8-10 m class ground-based telescopes, Spitzer-IRS, Herschel-PACS, future facilities such as the JWST, SOFIA, E-ELT, the Thirty Meter Telescope (TMT), and the Giant Magellan Telescope (GMT), in addition to (sub)millimeter facilities, such as ALMA. While not explicitly modeled in this paper, we also expect applications to future infrared interferometer facilities, such as VLT-MATISSE. We find that a primary reason that infrared molecular spectroscopy of disks in the $N$ band $\left(\mathrm{H}_{2} \mathrm{O}\right.$ and a wide range of other species) has not received much attention so far is due to a sensitivity deficit of roughly a factor of 10 . This will be remedied by the ELT generation and the JWST, and we predict that this will become a highly active research area in the future. The potential applications for RADLite reach beyond protoplanetary disks. Any axisymmetric structure can be modeled. This could potentially include protoplanetary nebulae, the atmospheres of asymptotic giant branch stars, and active galactic nuclei.

An anonymous referee provided a thorough and constructive report that improved the manuscript considerably. The authors are grateful to Sarah Kendrew, Leiden Observatory, for providing a high-resolution infrared transmission spectrum model. Discussions with Joan Najita, Colette Salyk, and Marco Spaans have been valuable during the preparation of the manuscript. Support for K.M.P. was provided by NASA through Hubble Fellowship grant \#01201.01 awarded by the Space Telescope Science Institute, which is operated by the Association of Universities for Research in Astronomy, Inc., for NASA, under contract NAS 5-26555. R.M. has been supported by NSF grant AST-0708922.

\section{REFERENCES}

Acke, B., van den Ancker, M. E., \& Dullemond, C. P. 2005, A\&A, 436, 209 Ayliffe, B. A., \& Bate, M. R. 2009, MNRAS, 393, 49

Blake, G. A., \& Boogert, A. C. A. 2004, ApJ, 606, L73

Brittain, S. D., Rettig, T. W., Simon, T., \& Kulesa, C. 2005, ApJ, 626, 283

Brittain, S. D., Simon, T., Najita, J. R., \& Rettig, T. W. 2007, ApJ, 659, 685

Brown, J. M., et al. 2007, ApJ, 664, L107

Calvet, N., D’Alessio, P., Hartmann, L., Wilner, D., Walsh, A., \& Sitko, M. 2002, ApJ, 568, 1008

Canup, R. M., \& Ward, W. R. 2002, AJ, 124, 3404

Carr, J. S., \& Najita, J. R. 2008, Science, 319, 1504

Chiang, E. I., \& Goldreich, P. 1997, ApJ, 490, 368
Dullemond, C. P., \& Dominik, C. 2004, A\&A, 417, 159

Dullemond, C. P., \& Turolla, R. 2000, A\&A, 360, 1187

Encrenaz, T. 2008, ARA\&A, 46, 57

Evans, N. J., II, et al. 2009, ApJS, 181, 321

Fedele, D., et al. 2008, A\&A, 491, 809

Gibb, E. L., Van Brunt, K. A., Brittain, S. D., \& Rettig, T. W. 2007, ApJ, 660, 1572

Glassgold, A. E., Meijerink, R., \& Najita, J. R. 2009, ApJ, 701, 142

Glassgold, A. E., Najita, J., \& Igea, J. 2004, ApJ, 615, 972

Gorti, U., \& Hollenbach, D. 2008, ApJ, 683, 287

Goto, M., Usuda, T., Dullemond, C. P., Henning, T., Linz, H., Stecklum, B., \& Suto, H. 2006, ApJ, 652, 758

Hartmann, L., Calvet, N., Gullbring, E., \& D’Alessio, P. 1998, ApJ, 495, 385

Hogerheijde, M. R., \& van der Tak, F. F. S. 2000, A\&A, 362, 697

Knez, C., Lacy, J. H., Evans, N. J., II, van Dishoeck, E. F., \& Richter, M. J. 2009, ApJ, 696, 471

Kurucz, R. L. 1993, VizieR Online Data Catalog, 6039, 0

Lada, C. J., et al. 2006, AJ, 131, 1574

Lahuis, F., van Dishoeck, E. F., Blake, G. A., Evans, N. J., II, Kessler-Silacci, J. E., \& Pontoppidan, K. M. 2007, ApJ, 665, 492

Lahuis, F., et al. 2006, ApJ, 636, L145

Levison, H. F., \& Morbidelli, A. 2003, Nature, 426, 419

Lopez, B., et al. 2008, Proc. SPIE, 7013, 70132B

Lyons, J. R., \& Young, E. D. 2005, Nature, 435, 317

Machida, M. N., Kokubo, E., Inutsuka, S.-i., \& Matsumoto, T. 2008, ApJ, 685, 1220

Meijerink, R., Poelman, D. R., Spaans, M., Tielens, A. G. G. M., \& Glassgold, A. E. 2008, ApJ, 689, L57

Meijerink, R., Pontoppidan, K. M., Blake, G. A., Poelman, D., \& Dullemond, C. P. 2009, ApJ, 704, 1471

Morbidelli, A., Bottke, Jr., W. F., Froeschlé, C., \& Michel, P. 2002, in Asteroids III, ed. W. F. Bottke Jr., A. Cellino, P. Paolicchi, \& R. P. Binzel (Tucson, AZ: Univ. Arizona Press), 409

Najita, J., Carr, J. S., \& Mathieu, R. D. 2003, ApJ, 589, 931

Najita, J. R., et al. 2009, ApJ, 697, 957

Pascucci, I., et al. 2007, ApJ, 663, 383

Pavlyuchenkov, Y., Semenov, D., Henning, T., Guilloteau, S., Piétu, V., Launhardt, R., \& Dutrey, A. 2007, ApJ, 669, 1262

Poelman, D. R., \& Spaans, M. 2005, A\&A, 440, 559

Pontoppidan, K. M., Blake, G. A., van Dishoeck, E. F., Smette, A., Ireland, M J., \& Brown, J. 2008, ApJ, 684, 1323

Pontoppidan, K. M., Dullemond, C. P., van Dishoeck, E. F., Blake, G. A., Boogert, A. C. A., Evans, N. J., II, Kessler-Silacci, J. E., \& Lahuis, F. 2005, ApJ, 622, 463

Rothman, L. S., et al. 2005, J. Quant. Spectrosc. Radiat. Transfer, 96, 139

Sabatke, E., Burge, J., \& Sabatke, D. 2005, Appl. Opt., 44, 1360

Salyk, C., Blake, G. A., Boogert, A. C. A., \& Brown, J. M. 2007, ApJ, 655 , L105

Salyk, C., Pontoppidan, K. M., Blake, G. A., Lahuis, F., van Dishoeck, E. F., \& Evans, N. J., II 2008, ApJ, 676, L49

Schöier, F. L., van der Tak, F. F. S., van Dishoeck, E. F., \& Black, J. H. 2005, A\&A, 432, 369

Semenov, D., Pavlyuchenkov, Y., Henning, T., Wolf, S., \& Launhardt, R. 2008, ApJ, 673, L195

Siess, L., Dufour, E., \& Forestini, M. 2000, A\&A, 358, 593

Smith, R., Pontoppidan, K. M., Young, E. D., Morris, M. R., \& van Dishoeck, E. F. 2009, ApJ, 701, 163

Strom, K. M., Strom, S. E., Edwards, S., Cabrit, S., \& Skrutskie, M. F. 1989, AJ, 97, 1451

Thi, W.-F., \& Bik, A. 2005, A\&A, 438, 557

Tsiganis, K., Gomes, R., Morbidelli, A., \& Levison, H. F. 2005, Nature, 435, 459

Udry, S., \& Santos, N. C. 2007, ARA\&A, 45, 397

van Boekel, R., Güdel, M., Henning, T., Lahuis, F., \& Pantin, E. 2009, A\&A, 497, 137

van der Plas, G., van den Ancker, M. E., Acke, B., Carmona, A., Dominik, C., Fedele, D., \& Waters, L. B. F. M. 2009, A\&A, 500, 1137

van der Plas, G., van den Ancker, M. E., Fedele, D., Acke, B., Dominik, C., Waters, L. B. F. M., \& Bouwman, J. 2008, A\&A, 485, 487

van Zadelhoff, G.-J., van Dishoeck, E. F., Thi, W.-F., \& Blake, G. A. 2001, A\&A, 377,566

Woitke, P., Kamp, I., \& Thi, W.-F. 2009, A\&A, 501, 383

Wolf, S., \& D'Angelo, G. 2005, ApJ, 619, 1114

Wooden, D., Desch, S., Harker, D., Gail, H.-P., \& Keller, L. 2007, in Protostars and Planets V, ed. B. Reipurth, D. Jewitt, \& K. Keil (Tucson, AZ: Univ. Arizona Press), 815

Woods, P. M., \& Willacy, K. 2009, ApJ, 693, 1360 